\title{
INTEGER MATRIX EXACT COVERING SYSTEMS AND PRODUCT IDENTITIES FOR THETA FUNCTIONS
}

\author{
ZHU CAO
}

\begin{abstract}
In this paper, we prove that there is a natural correspondence between product identities for theta functions and integer matrix exact covering systems. We show that since $\mathbb{Z}^{n}$ can be taken as the disjoint union of a lattice generated by $n$ linearly independent vectors in $\mathbb{Z}^{n}$ and a finite number of its translates, certain products of theta functions can be written as linear combinations of other products of theta functions. We firstly give a general theorem to write a product of $n$ theta functions as a linear combination of other products of theta functions. Many known identities for products of theta functions are shown to be special cases of our main theorem. Several entries in Ramanujan's notebooks as well as new identities are proved as applications, including theorems for products of three and four theta functions that have not been obtained by other methods.
\end{abstract}

\section{Introduction and Main Theorem}

We use the standard notation for $q$-products, defining

$$
(a)_{\infty}:=(a ; q)_{\infty}=\prod_{k=0}^{\infty}\left(1-a q^{k}\right), \quad|q|<1 .
$$

The celebrated Jacobi triple product identity is given by [4, p. 10]

$$
\sum_{n=-\infty}^{\infty} q^{n^{2}} z^{n}=\left(-q z ; q^{2}\right)_{\infty}\left(-q / z ; q^{2}\right)_{\infty}\left(q^{2} ; q^{2}\right)_{\infty}, \quad|q|<1 .
$$

We define the modified Jacobi theta function $\langle x ; q\rangle_{\infty}:=(q ; q)_{\infty}(x ; q)_{\infty}(q / x ; q)_{\infty}$. So the Jacobi triple product identity can be written as

$$
\sum_{n=-\infty}^{\infty} q^{n^{2}} z^{n}=\left\langle-q z ; q^{2}\right\rangle_{\infty}, \quad|q|<1
$$

We use abbreviated forms for $q$-products

$$
\begin{aligned}
&(\alpha, \beta, \ldots, \gamma ; q)_{\infty}::=(\alpha ; q)_{\infty}(\beta ; q)_{\infty} \ldots(\gamma ; q)_{\infty}, \\
&\langle\alpha, \beta, \ldots, \gamma ; q\rangle_{\infty}:=\langle\alpha ; q\rangle_{\infty}\langle\beta ; q\rangle_{\infty} \ldots\langle\gamma ; q\rangle_{\infty} .
\end{aligned}
$$

Ramanujan's general theta function is defined as

$$
f(a, b):=\sum_{n=-\infty}^{\infty} a^{n(n+1) / 2} b^{n(n-1) / 2},|a b|<1 .
$$


By Jacobi's triple product identity, we have

$$
f(a, b)=(-a ; a b)_{\infty}(-b ; a b)_{\infty}(a b ; a b)_{\infty} .
$$

It is easy to verify that

$$
\begin{aligned}
f(a, b) & =f(b, a), \\
f(1, a) & =2 f\left(a, a^{3}\right), \\
f(-1, a) & =0,
\end{aligned}
$$

and, if $n$ is an integer,

$$
f(a, b)=a^{n(n+1) / 2} b^{n(n-1) / 2} f\left(a(a b)^{n}, b(a b)^{-n}\right) .
$$

The three most important cases of $f(a, b)$, namely

$$
\begin{aligned}
\varphi(q) & :=\sum_{n=-\infty}^{\infty} q^{n^{2}}=f(q, q)=\left(-q ; q^{2}\right)_{\infty}^{2}\left(q^{2} ; q^{2}\right)_{\infty}, \\
\psi(q) & :=\sum_{n=0}^{\infty} q^{n(n+1) / 2}=f\left(q, q^{3}\right)=\frac{\left(q^{2} ; q^{2}\right)_{\infty}}{\left(q ; q^{2}\right)_{\infty}} \\
f(-q) & :=f\left(-q,-q^{2}\right)=(q ; q)_{\infty},
\end{aligned}
$$

are also used throughout this paper. We also define $\chi(q)=\left(-q ; q^{2}\right)_{\infty}$, which is not a theta function. We define it mainly for convenience.

Define $(a)_{n}:=a(a+1)(a+2) \cdots(a+n-1)$. A modular equation of degree $n$ is an algebraic relation of $\alpha$ and $\beta$ which is induced by the relation

$$
\frac{{ }_{2} F_{1}\left(\frac{1}{2}, \frac{1}{2} ; 1 ; 1-\beta\right)}{{ }_{2} F_{1}\left(\frac{1}{2}, \frac{1}{2} ; 1 ; \beta\right)}=n \frac{{ }_{2} F_{1}\left(\frac{1}{2}, \frac{1}{2} ; 1 ; 1-\alpha\right)}{{ }_{2} F_{1}\left(\frac{1}{2}, \frac{1}{2} ; 1 ; \alpha\right)},
$$

where

$$
{ }_{2} F_{1}(a, b ; c ; z):=\sum_{n=0}^{\infty} \frac{(a)_{n}(b)_{n}}{(c)_{n} n !} z^{n}, \quad|z|<1,
$$

is the Gaussian or ordinary hypergeometric functions. When (1.8) holds, we say that $\beta$ has degree $n$ over $\alpha$.

A system of congruences $a_{i}\left(\bmod n_{i}\right)$ with $1 \leq i \leq k$ is called a covering system (or complete residue system) if every integer $y$ satisfies $y \equiv a_{i}\left(\bmod n_{i}\right)$ for at least one value of $i$.

A covering system in which each integer is covered by just one congruence is called an exact covering system (ECS). In other words, an exact covering system is a partition of the integers into a finite set of arithmetic sequences.

Corresponding to the exact covering system $\{r(\bmod k)\}_{r=0}^{k-1}$, we can write any theta function as the linear combination of $k$ theta functions

$$
f(a, b)=\sum_{n=-\infty}^{\infty} a^{n(n+1) / 2} b^{n(n-1) / 2}=\sum_{r=0}^{k-1} \sum_{n=-\infty}^{\infty} a^{(k n+r)(k n+r+1) / 2} b^{(k n+r)(k n+r-1) / 2}
$$


PRODUCT IDENTITIES FOR THETA FUNCTIONS

$$
=\sum_{r=0}^{k-1} a^{r(r+1) / 2} b^{r(r-1) / 2} f\left(a^{k(k+1) / 2+k r} b^{k(k-1) / 2+k r}, a^{k(k-1) / 2-k r} b^{k(k+1) / 2-k r}\right) .
$$

If we define $U_{k}=a^{k(k+1) / 2} b^{k(k-1) / 2}, V_{k}=a^{k(k-1) / 2} b^{k(k+1) / 2}$, then we can write (1.9) as

$$
f(a, b)=f\left(U_{1}, V_{1}\right)=\sum_{r=0}^{k-1} U_{r} f\left(\frac{U_{k+r}}{U_{r}}, \frac{V_{k-r}}{U_{r}}\right),
$$

This is [2, pp. 48-49, Entry 31]. Letting $k=2$ in (1.10), we have

$$
f(a, b)=f\left(a^{3} b, a b^{3}\right)+a f\left(b / a, a^{5} b^{3}\right),
$$

which is used in this paper.

A natural question is: For a product of $n(n \geq 2)$ theta functions, do we have similar results as (1.10)? In other words, can we write a product of $n$ theta functions as a linear combination of other products of theta functions? The quintuple product identity and the septuple product identity are examples for products of two theta functions.

Theorem 1.1 (The Quintuple Product Identity). For $a \neq 0$,

$$
\begin{aligned}
& (-a q ; q)_{\infty}(-1 / a ; q)_{\infty}\left(a^{2} q ; q^{2}\right)_{\infty}\left(q / a^{2} ; q^{2}\right)_{\infty}(q ; q)_{\infty} \\
= & \left(a^{3} q^{2} ; q^{3}\right)_{\infty}\left(q / a^{3} ; q^{3}\right)_{\infty}\left(q^{3} ; q^{3}\right)_{\infty}+a^{-1}\left(a^{3} q ; q^{3}\right)_{\infty}\left(q^{2} / a^{3} ; q^{3}\right)_{\infty}\left(q^{3} ; q^{3}\right)_{\infty} .
\end{aligned}
$$

Theorem 1.2 (The Septuple Product Identity). For $a \neq 0$,

$$
\begin{aligned}
& \left\langle a ; q^{2}\right\rangle_{\infty}\left\langle a^{2} ; q^{2}\right\rangle_{\infty} \\
= & \left\langle q^{4} ; q^{10}\right\rangle_{\infty}\left[\left\langle a^{5} q^{2} ; q^{10}\right\rangle_{\infty}+a^{3}\left\langle a^{5} q^{8} ; q^{10}\right\rangle_{\infty}\right]-\left\langle q^{2} ; q^{10}\right\rangle_{\infty}\left[a\left\langle a^{5} q^{4} ; q^{10}\right\rangle_{\infty}+a^{2}\left\langle a^{5} q^{6} ; q^{10}\right\rangle_{\infty}\right] .
\end{aligned}
$$

For the history of the quintuple product identity, readers can refer to S. Cooper's survey [16]. The septuple product identity was first discovered by M. D. Hirschhorn [21, (3.1)] in 1983 .

Winquist's identity is an example for products of four theta functions.

Theorem 1.3 (Winquist's Identity). For any nonzero complex numbers $a, b$,

$$
\begin{aligned}
& \sum_{m=-\infty}^{\infty} \sum_{n=-\infty}^{\infty}(-1)^{m+n} q^{\frac{3 m^{2}+3 n^{2}+3 m+n}{2}}\left(a^{-3 m} b^{-3 n}-a^{-3 m} b^{3 n+1}-a^{-3 n+1} b^{-3 m-1}+a^{3 n+2} b^{-3 m-1}\right) \\
= & (a, q / a, b, q / b, a b, q / a b, a / b, b q / a, q, q ; q)_{\infty} .
\end{aligned}
$$

Winquist's identity plays a vital role in the first elementary proof of Ramanujan's congruence $p(11 n+6) \equiv 0(\bmod 11)$ given by L. Winquist in [34], where $p(n)$ denote the partition function. Readers can refer to its latest proof given by S. H. Chan [11] for its brief history.

In [22, Hirschhorn obtained a generalization of Winquist's identity by multiplying four triple products. He considered a very nice transformation matrix and derived a four-parameter identity which gives Winquist's identity as a special case. Inspired by Hirschhorn's work, S.-S. Huang [25] showed that Hirschhorn's generalization of the quintuple product identity [23] can also be obtained by using the idea in [22]. Other 
cases of this kind of transformation are discussed in [3, pp. 190-191], [20, and [1]. But all of the above are just special cases; there have been few systematical study on the conditions under which the product of two or more theta functions can be written as the linear combination of other products of theta functions.

In this paper, we consider a class of "generalized orthogonal" transformation matrices and give a systematic approach for obtaining product identities. Although we give the first general theorem on the product of any $n$ theta functions, most of the examples discussed in this paper are for products of two theta functions. Our main theorem for products of two theta functions is a generalization of the Schröter formula. Many known identities for products of two theta functions, including M. D. Hirschhorn's generalization of the quintuple product identity, the septuple product identity, some modular relations for the Göllnitz-Gordon functions found by S.-S. Huang in [24, identities involving septic Rogers-Ramanujan functions obtained by H. Hahn [20], a general theorem by W. Chu and Q. Yan [14], and the Blecksmith-Brillhart-Gerst theorem [6], are shown to be special cases of our main theorem. We also derive several new theorems for products of three or more theta functions as applications, including an analogue of Winquist's identity and a new representation of $(q ; q)_{\infty}^{8}$.

Let $l_{i} \in \mathbb{Z}^{+}, a_{i} b_{i}=q^{l_{i}},(i=1,2, \ldots, n)$. Without lose of generality, suppose $l_{1} \leq$ $l_{2} \cdots \leq l_{n}$. We consider a product of $n$ theta functions

$$
S:=\prod_{i=1}^{n} f\left(a_{i}, b_{i}\right)=\sum_{x_{1}, x_{2} \cdots, x_{n}=-\infty}^{\infty} a_{1}^{\frac{x_{1}^{2}+x_{1}}{2}} b_{1}^{\frac{x_{1}^{2}-x_{1}}{2}} \cdots a_{n} \frac{x_{n}^{2}+x_{n}}{2} b_{n} \frac{x_{n}^{2}-x_{n}}{2} .
$$

Next, we change the variables from $x_{i}$ to $y_{i}(i=1,2, \ldots, n)$ by the transformation $y=A x$, where $A$ is an integer matrix with $\operatorname{det} A \neq 0$. Set

$$
x=\left(\begin{array}{c}
x_{1} \\
x_{2} \\
\vdots \\
x_{n}
\end{array}\right), \quad y=\left(\begin{array}{c}
y_{1} \\
y_{2} \\
\vdots \\
y_{n}
\end{array}\right) .
$$

By the inverse formula,

$$
x=A^{-1} y=\frac{1}{\operatorname{det} A} A^{*} y,
$$

where $A^{*}$ is the adjugate of $A$.

For $k \in \mathbb{Z}, 1 \leq k \leq n$, the $k$ th determinantal divisor of $A$, denoted by $d_{k}(A)$, is defined as the greatest common divisor of all the $k$ by $k$ determinantal minors of $A$. It is easy to see that $d_{n}=\operatorname{det} A$. We let $d_{0}=1$ for convenience. Note that $d_{k} \mid d_{k+1}$. The invariant factors of $A$ are defined as

$$
S_{k}(A)=\frac{d_{k}}{d_{k-1}}
$$

By the Smith normal form theorem, we have $\operatorname{det} A=s_{1} \cdots s_{n}$. So

$$
\frac{\operatorname{det} A}{d_{n-1}(A)}=s_{n}(A) \text {. }
$$


From (1.17), we can rewrite (1.16) as

$$
x=\frac{1}{s_{n}(A)} \cdot \frac{A^{*}}{d_{n-1}(A)} y,
$$

where $\frac{A^{*}}{d_{n-1}(A)}$ is an integer matrix. Letting $\operatorname{sgn}\left(s_{n}(A)\right) \frac{A^{*}}{d_{n-1}(A)}=B,\left|s_{n}(A)\right|=d$, we can rewrite (1.18) as

$$
x=\frac{1}{d} B y .
$$

So we have

$$
B y \equiv 0 \quad(\bmod d)
$$

We want to write (1.15) as a linear combination of products of other theta functions. After replacing the variables $x_{1}, x_{2}, \ldots, x_{n}$ with $y_{1}, y_{2}, \ldots, y_{n}$, we have the restriction (1.20) regarding $y$. So $y_{i}$ cannot take all the integer values unless $d=1$. The system of homogeneous congruences (1.20) is always consistent since $y \equiv 0(\bmod d)$ is a solution. Suppose we have $k$ solutions to (1.20).

Reduced case: $y \equiv 0(\bmod d)$. We can replace $y$ by $d y$ in $(1.19)$. So we have $x=B y$. Replacing $x$ with $B y$ in the right-hand side of (1.15), we obtain the contribution of this case to $S$

$$
\begin{aligned}
\sum_{y_{1}, \cdots, y_{n}=-\infty}^{\infty} & a_{1} \frac{\left(b_{11} y_{1}+b_{12} y_{2}+\cdots+b_{1 n} y_{n}\right)^{2}+\left(b_{11} y_{1}+b_{12} y_{2}+\cdots+b_{1 n} y_{n}\right)}{2} b_{1} \frac{\left(b_{11} y_{1}+b_{12} y_{2}+\cdots+b_{1 n} y_{n}\right)^{2}-\left(b_{11} y_{1}+b_{12} y_{2}+\cdots+b_{1 n} y_{n}\right)}{2} \\
& \cdots a_{n} \frac{\left(b_{n 1} y_{1}+b_{n 2} y_{2}+\cdots+b_{n n} y_{n}\right)^{2}+\left(b_{n 1} y_{1}+b_{n 2} y_{2}+\cdots+b_{n n} y_{n}\right)}{2} b_{n} \frac{\left(b_{n 1} y_{1}+b_{n 2} y_{2}+\cdots+b_{n n} y_{n}\right)^{2}-\left(b_{n 1} y_{1}+b_{n 2} y_{2}+\cdots+b_{n n} y_{n}\right)}{2} .
\end{aligned}
$$

We need the coefficients of all $y_{i} y_{j}=0(i \neq j, i, j=1,2, \ldots, n)$ in order to separate $y_{1}, y_{2}, \ldots, y_{n}$ and write $S$ as a linear combination of products of theta functions. So we have the requirements

$$
\left\{\begin{array}{rcrcrl}
l_{1} b_{11} b_{12} & + & l_{2} b_{21} b_{22} & +\cdots+ & l_{n} b_{n 1} b_{n 2}= & 0 \\
l_{1} b_{11} b_{13} & + & l_{2} b_{21} b_{23} & +\cdots+ & l_{n} b_{n 1} b_{n 3}= & 0 \\
\vdots & \vdots & & \vdots & \vdots & \\
l_{1} b_{1(n-1)} b_{1 n} & + & l_{2} b_{2(n-1)} b_{2 n} & +\cdots+ & l_{n} b_{n(n-1)} b_{n n}= & 0 .
\end{array}\right.
$$

By Jacobi's triple product identity (1.2), now we can rewrite (1.21) as a product of $n$ theta functions

$$
\begin{aligned}
& f\left(a_{1} \frac{b_{11}^{2}+b_{11}}{2} b_{1} \frac{b_{11}^{2}-b_{11}}{2} \cdots a_{n} \frac{b_{n 1}^{2}+b_{n 1}}{2} b_{n} \frac{b_{n 1}^{2}-b_{n 1}}{2}, a_{1} \frac{b_{11}^{2}-b_{11}}{2} b_{1} \frac{b_{11}^{2}+b_{11}}{2} \cdots a_{n} \frac{b_{n 1}^{2}-b_{n 1}}{2} b_{n} \frac{b_{n 1}^{2}+b_{n 1}}{2}\right) \cdots \\
& \times f\left(a_{1} \frac{b_{1 n}^{2}+b_{1 n}}{2} b_{1} \frac{b_{1 n}^{2}-b_{1 n}}{2} \cdots a_{n} \frac{b_{n n}^{2}+b_{n n}}{2} b_{n} \frac{b_{n n}^{2}-b_{n n}}{2}, a_{1} \frac{b_{1 n}^{2}-b_{1 n}}{2} b_{1} \frac{b_{1 n}^{2}+b_{1 n}}{2} \cdots a_{n} \frac{b_{n n}^{2}-b_{n n}}{2} b_{n} \frac{b_{n n}^{2}+b_{n n}}{2}\right) .
\end{aligned}
$$

General case: If $y \equiv c_{r}(\bmod d)(r=0,1,2, \ldots, k-1)$ is a solution of (1.20), we substitute $y$ with $d y+c_{r}$ in (1.19). Then we have $x=B y+\frac{1}{d} B c_{r}$, where $\frac{1}{d} B c_{r}$ is an $n$-dimensional integer vector. By adding the contribution of each solution to the sum $S$, we can write $S$ as a linear combination of products of theta functions. We need $(1.22)$ in all cases in order to separate $y_{1}, y_{2}, \ldots, y_{n}$. 
We can find that in the first theta function in (1.23),

$$
\begin{aligned}
& a_{1} \frac{b_{11}^{2}+b_{11}}{2} b_{1} \frac{b_{11}^{2}-b_{11}}{2} \cdots a_{n} \frac{b_{n 1}^{2}+b_{n 1}}{2} b_{n} \frac{b_{n 1}^{2}-b_{n 1}}{2} \times a_{1} \frac{b_{11}^{2}-b_{11}}{2} b_{1} \frac{b_{11}^{2}+b_{11}}{2} \cdots a_{n} \frac{\frac{b_{n 1}^{2}-b_{n 1}}{2}}{2} b_{n} \frac{b_{n 1}^{2}+b_{n 1}}{2} \\
= & \left(a_{1} b_{1}\right)^{b_{11}{ }^{2}} \cdots\left(a_{n} b_{n}\right)^{b_{n 1}{ }^{2}}=q^{l_{1} b_{11}{ }^{2}+\cdots+l_{n} b_{n 1}{ }^{2}} .
\end{aligned}
$$

Similarly we can find that the rest of the products in (1.23) are $q^{l_{1} b_{12}{ }^{2}+\cdots+l_{n} b_{n 2}{ }^{2}}, \cdots$, $q^{l_{1} b_{1 n}{ }^{2}+\cdots+l_{n} b_{n n}{ }^{2}}$. It can be shown that this pattern holds for any part in the linear combination. It is very useful in finding the theta functions in the linear combination and is illustrated later in the proof of (2.46).

Let $B=\left(\mathbf{b}_{\mathbf{1}}, \ldots, \mathbf{b}_{\mathbf{n}}\right)$, where $\mathbf{b}_{\mathbf{j}}$ is the $j$ th column of $B$. If we choose integers $l_{1}=l_{2}=\cdots=l_{n}$, then (1.22) implies that $\left\{\mathbf{b}_{\mathbf{1}}, \ldots, \mathbf{b}_{\mathbf{n}}\right\}$ is an orthogonal set. If the set of all the columns of a matrix $B$ is an orthogonal set, then $B^{T} B$ is a diagonal matrix with all entries positive integers on the main diagonal. We define

$$
D=\left(\begin{array}{ccccc}
l_{1} & 0 & 0 & \cdots & 0 \\
0 & l_{2} & 0 & \cdots & 0 \\
0 & 0 & l_{3} & \cdots & 0 \\
\vdots & \vdots & \vdots & \ddots & \vdots \\
0 & 0 & 0 & \cdots & l_{n}
\end{array}\right)
$$

where $l_{1}, l_{2}, \ldots, l_{n}$ are positive integers. Then (1.22) implies that $B^{T} D B$ is a diagonal matrix with all entries positive integers on the main diagonal. So the set of all the columns of $B$ is a kind of "generalized orthogonal" set. For fixed $l_{i}(i=1,2, \ldots, n)$, there are infinitely many solutions $b_{i j}(i, j=1,2, \ldots, n)$ to the system of equations (1.22). So product of any $n$ theta functions can be written as the linear combinations of other products of theta functions, not in unique way.

We have $\operatorname{det} A^{*}=(\operatorname{det} A)^{n-1}$. Since $A \cdot A^{*}=\operatorname{det} A \cdot I$, where $A$ is an integer matrix, we require $(\operatorname{det} A) \cdot\left(A^{*}\right)^{-1}$ to be an integer matrix.

Without lose of generality, we have the following procedure for obtaining seriesproduct identities.

1. For fixed positive integers $l_{1}, l_{2}, \ldots, l_{n}$, find all $n \times n$ matrices $B^{\prime}$ satisfying the "generalized orthogonal" relation (1.22), where $\operatorname{det} B^{\prime}= \pm\left(d^{\prime}\right)^{n-1}$ and $d^{\prime} B^{\prime-1}$ is an integer matrix, where $d^{\prime} \in N$.

2. For the system of congruences $B^{\prime} y \equiv 0\left(\bmod d^{\prime}\right)$ obtained from step 1 , we divide by the greatest common divisor on both sides and rewrite it as $B y \equiv 0(\bmod d)$. Next we solve the system of congruences $B y \equiv 0(\bmod d)$. Suppose we have $k$ solutions. By computing the contribution of each solution, we can write each product of $n$ theta functions as the linear combination of $k$ products of $n$ theta functions.

If $y \equiv c_{r}(\bmod d)(r=0,1,2, \ldots, k-1)$ is the solution set of (1.20), we substitute each $y$ with $d y+c_{r}$ in (1.19). Then we have $x=B y+\frac{1}{d} B c_{r},(r=0,1,2, \ldots, k-1)$. We assume $c_{0}=0$. It is easy to see that for $y \in \mathbb{Z}^{n},\left\{B y+\frac{1}{d} B c_{r}\right\}_{r=0}^{k-1}$ covers $\mathbb{Z}^{n}$ and there is no overlap between the members. By defining the binary operation as vector addition in the abelian additive group $\mathbb{Z}^{n}, B \mathbb{Z}^{n} \triangleleft \mathbb{Z}^{n}$, and two vectors $\mathbf{a} \equiv \mathbf{b}$ if and only if $\mathbf{a}-\mathbf{b} \in B \mathbb{Z}^{n}$. So now we can take $\left\{B \mathbb{Z}^{n}+\frac{1}{d} B c_{r}\right\}_{r=0}^{k-1}$ as an integer matrix exact covering system of $\mathbb{Z}^{n}$. In other words, a partition of $\mathbb{Z}^{n}$ into a lattice 
and a finite number of its translates. If we view this geometrically, we are counting the distinct points inside the $n$-dimensional parallelotope spanned by the columns of $B$ (Two points are counted as one if the difference of the corresponding vector is a linear combination of the columns of $B$ ). From M. A. Fiol [18, Proposition 2], the number of equivalence classes in the quotient group is $|\operatorname{det} B|$. So $k=|\operatorname{det} B|$. We know that $|\operatorname{det} B|=$ volume of the parallelotope spanned by the columns of $B$. So this implies that numerically the number of distinct points inside a parallelotope equals the volume of the parallelotope.

So now it is clear that we are really looking for a special kind of ECS of $\mathbb{Z}^{n}$ corresponds to "generalized orthogonal" matrix $B$. Although we can find the ECS by solving the system of congruences (1.20), there is no general formula for the solution set of system of congruences. A better approach is to find the congruence classes by finding $k$ distinct points in $\mathbb{Z}^{n}$ if $B$ is known with $|\operatorname{det} B|=k$. We have the same covering system if we multiply a column of $B$ by -1 , or we interchange two columns of $B$. So here we can assume $\operatorname{det} B>0$.

By choosing special coset representatives, we give the main theorem for products of $n$ theta functions.

Theorem 1.4. Let $l_{i} \in \mathbb{Z}^{+}, a_{i} b_{i}=q^{l_{i}},(i=1,2, \ldots, n)$. Let $B=\left(b_{i j}\right)_{n \times n}$ be an invertible integer matrix satisfying (1.22). Let $k=\operatorname{det} B>0$. Let $B^{*}$ be the adjugate of $B$. At least one of the entries of $B^{*}$ has no common factor with $k$. We suppose an entry in the $j$ th column of $B^{*}$ is coprime to $k$. Then

$$
\begin{aligned}
& \prod_{i=1}^{n} f\left(a_{i}, b_{i}\right)
\end{aligned}
$$

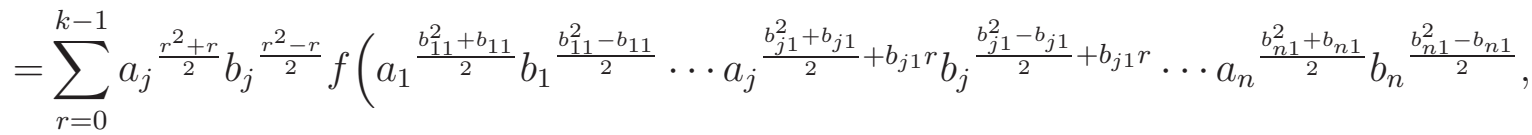

$$
\begin{aligned}
& \left.a_{1} \frac{b_{11}^{2}-b_{11}}{2} b_{1} \frac{b_{11}^{2}+b_{11}}{2} \cdots a_{j} \frac{b_{j 1}^{2}-b_{j 1}}{2}-b_{j 1} r b_{j} \frac{b_{j 1}^{2}+b_{j 1}}{2}-b_{j 1} r \cdots a_{n} \frac{\frac{b_{n 1}^{2}-b_{n 1}}{2}}{2} b_{n} \frac{b_{n 1}^{2}+b_{n 1}}{2}\right) \cdots
\end{aligned}
$$

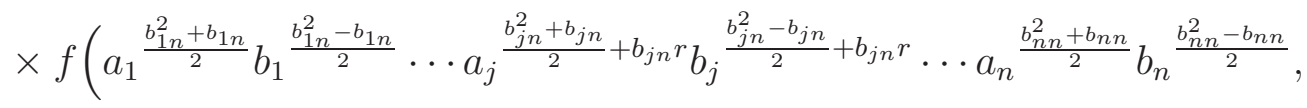

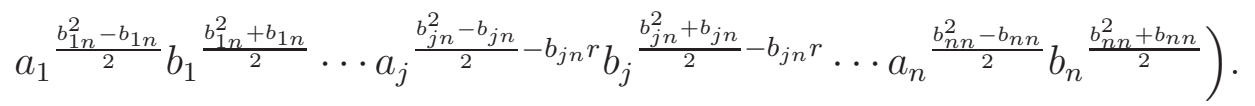

Remark 1.1. The above theorem remains valid if the sum over $r$ runs over a complete residue system $(\bmod k)$. For instance, $\left[-\frac{k}{2}\right]+1 \leq r \leq\left[\frac{k}{2}\right]$.

Proof. For (1.24), we only need to show that for integer matrix exact covering system

$$
\left\{B \mathbb{Z}^{n}+\left(\begin{array}{c}
0 \\
\vdots \\
r \\
\vdots \\
0
\end{array}\right)\right\}_{r=0}^{k-1}
$$


where $r$ is in the $j$ th row, there is no overlap between the members of the covering system. That is, $B y=\left(\begin{array}{c}0 \\ \vdots \\ r \\ \vdots \\ 0\end{array}\right),(r=1,2, \ldots, k-1)$ has no integer vector solutions. From $B y=\left(\begin{array}{c}0 \\ \vdots \\ r \\ \vdots \\ 0\end{array}\right)$, by the inverse formula, we have

$$
y=\frac{1}{k} B^{*}\left(\begin{array}{c}
0 \\
\vdots \\
r \\
\vdots \\
0
\end{array}\right)=\frac{1}{k}\left(\begin{array}{c}
b_{1 j}^{*} r \\
b_{2 j}^{*} r \\
\vdots \\
b_{n j}^{*} r
\end{array}\right)
$$

Since an entry in the $j$ th column of $B^{*}$ is coprime to $k$, at least one entry of $\left(\begin{array}{c}b_{1 j}^{*} r \\ b_{2 j}^{*} r \\ \vdots \\ b_{n j}^{*} r\end{array}\right)$ is not divisible by $k$. So $B y=\left(\begin{array}{c}0 \\ \vdots \\ r \\ \vdots \\ 0\end{array}\right),(r=1,2, \ldots, k-1)$ has no integer vector solution. We finish the proof.

Theorem 1.24 is symmetric with respect to $a_{i}$ and $b_{i}(i=1,2, \cdots, n)$. Interchanging the positions of $a_{i}$ and $b_{i}$ is equivalent to multiple by -1 on the $i$ th row of $B$.

Corollary 1.1. In Theorem 2.1, if we further require that for any column of B, the sum of all the entries is an even number, then

$$
\begin{aligned}
& \prod_{i=1}^{n} f\left(a_{i}, b_{i}\right)+\prod_{i=1}^{n} f\left(-a_{i},-b_{i}\right)
\end{aligned}
$$

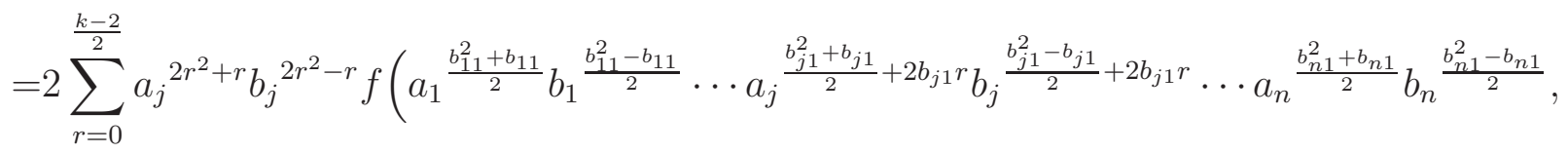

$$
\begin{aligned}
& \left.a_{1} \frac{b_{11}^{2}-b_{11}}{2} b_{1} \frac{b_{11}^{2}+b_{11}}{2} \cdots a_{j}^{\frac{b_{j 1}^{2}-b_{j 1}}{2}-2 b_{j 1} r} b_{j} \frac{b_{j 1}^{2}+b_{j 1}}{2}-2 b_{j_{1} 1} r \cdots a_{n}^{\frac{b_{n 1}^{2}-b_{n 1}}{2}} b_{n} \frac{b_{n 1}^{2}+b_{n 1}}{2}\right) \cdots
\end{aligned}
$$




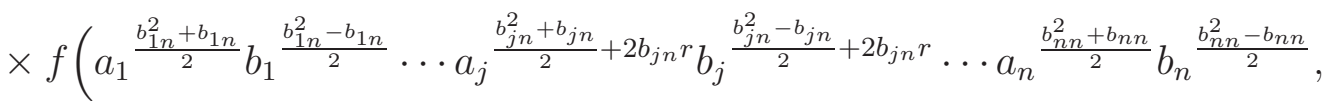

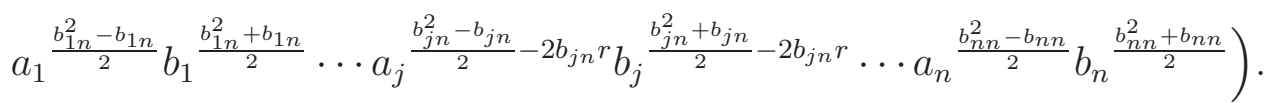

$$
\begin{aligned}
& \prod_{i=1}^{n} f\left(a_{i}, b_{i}\right)-\prod_{i=1}^{n} f\left(-a_{i},-b_{i}\right) \\
& =2 \sum_{r=0}^{\frac{k-2}{2}} a_{j}^{2 r^{2}+3 r+1} b_{j}{ }^{2 r^{2}+r}
\end{aligned}
$$

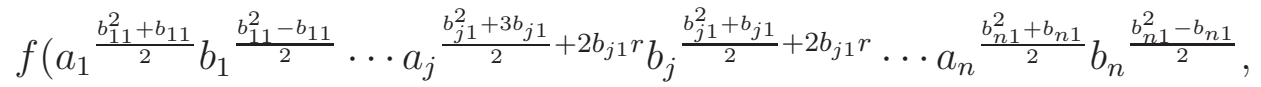

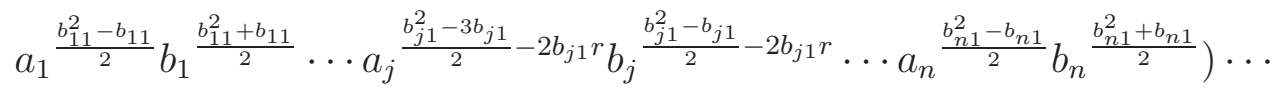

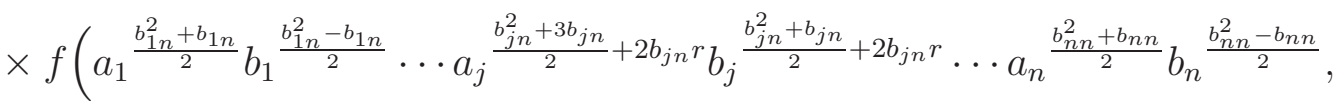

$$
\begin{aligned}
& \left.a_{1} \frac{b_{1 n}^{2}-b_{1 n}}{2} b_{1} \frac{b_{1 n}^{2}+b_{1 n}}{2} \cdots a_{j} \frac{b_{j n}^{2}-3 b_{j n}}{2}-2 b_{j n} r b_{j} \frac{b_{j n}^{2}-b_{j n}}{2}-2 b_{j n} r \cdots a_{n}^{\frac{b_{n n}^{2}-b_{n n}}{2}} b_{n}^{\frac{b_{n n}^{2}+b_{n n}}{2}}\right) .
\end{aligned}
$$

Proof. Since for any column of $B$, the sum of all the entries is an even number, it is easy to see that $k$ is even, and all of $b_{1 j}{ }^{2}+\cdots b_{n j}{ }^{2}(j=1,2, \cdots, n)$ are even. Replacing $a_{i}$ with $-a_{i}, b_{i}$ with $-b_{i}$ in (1.24), adding it to (1.24), and finally replacing $2 r$ with $r$, we obtain (1.25). The proof of (1.26) is similar.

Next we give an estimate of $\operatorname{det} B$ if all the entries of $B$ are nonzero. We have

$$
\begin{aligned}
& B^{T} D B= \\
& \left(\begin{array}{ccccc}
l_{1} b_{11}^{2}+l_{2} b_{21}^{2}+\cdots+l_{n} b_{n 1}^{2} & 0 & 0 & \cdots & 0 \\
0 & l_{1} b_{12}^{2}+l_{2} b_{22}^{2}+\cdots+l_{n} b_{n 2}^{2} & 0 & \cdots & 0 \\
\vdots & \vdots & \vdots & \ddots & \vdots \\
0 & 0 & 0 & \cdots & l_{1} b_{1 n}^{2}+l_{2} b_{2 n}^{2}+\cdots+l_{n} b_{n n}^{2}
\end{array}\right) .
\end{aligned}
$$

We take the determinant of both sides of (1.27) if all the entries of $B$ are nonzero and obtain

$$
\begin{aligned}
& (\operatorname{det} B)^{2} \cdot l_{1} l_{2} \cdots l_{n} \\
= & \left(l_{1} b_{11}^{2}+l_{2} b_{21}^{2}+\cdots+l_{n} b_{n 1}^{2}\right)\left(l_{1} b_{12}^{2}+l_{2} b_{22}^{2}+\cdots+l_{2} b_{n 2}^{2}\right) \cdots\left(l_{1} b_{1 n}^{2}+l_{2} b_{2 n}^{2}+\cdots+l_{n} b_{n n}^{2}\right) .
\end{aligned}
$$

So

$$
\operatorname{det} B=\sqrt{\frac{\left(l_{1} b_{11}^{2}+l_{2} b_{21}^{2}+\cdots+l_{n} b_{n 1}^{2}\right) \cdots\left(l_{1} b_{1 n}^{2}+l_{2} b_{2 n}^{2}+\cdots+l_{n} b_{n n}^{2}\right)}{l_{1} l_{2} \cdots l_{n}}} .
$$

We have

$$
l_{1} b_{1 i}^{2}+l_{2} b_{2 i}^{2}+\cdots+l_{n} b_{n i}^{2} \geq n\left(l_{1} b_{1 i}^{2} l_{2} b_{2 i}^{2} \cdots l_{n} b_{n i}^{2}\right)^{\frac{1}{n}} \quad(i=1,2, \ldots, n) .
$$


By (1.28) and (1.29), if we denote the product of all entries of the matrix $B$ by $\triangle B$, then we can obtain

$$
\operatorname{det} B \geq n^{\frac{n}{2}}(\triangle B)^{\frac{1}{n}}
$$

In order to minimize the number of solutions of the system of congruences (1.20), we need to find "generalized orthogonal" matrices with absolute values of determinants as small as possible. All the 3 by 3 and 4 by 4 matrices in this paper have entries chosen from $-2,-1,0,1,2$.

\section{Products of Two Theta Functions}

For a product of two theta functions $f(a, b) f(c, d)$ with $a b=q^{l_{1}}$ and $c d=q^{l_{2}}$, we need to find 2 by 2 invertible integer matrices $B$ satisfying the generalized orthogonal relation $l_{1} b_{11} b_{12}+l_{2} b_{21} b_{22}=0$. Without lose of generality, we can assume that three of $b_{11}, b_{12}, b_{21}, b_{22}$ are positive, one is negative. Now we give the following theorem for product of two theta functions, which is a special case of Theorem 1.4.

Theorem 2.1. Let $a b=q^{l_{1}}$ and $c d=q^{l_{2}}, l_{i} \in \mathbb{Z}^{+}$, where $i=1,2$, and let $B=\left(b_{i j}\right)$ be a $2 \times 2$ invertible integer matrix such that $l_{1} b_{11} b_{12}+l_{2} b_{21} b_{22}=0, \operatorname{gcd}\left(b_{21}, b_{11} b_{22}\right)=1$ or $\operatorname{gcd}\left(b_{22}, b_{12} b_{21}\right)=1$. Let $k=\operatorname{det} B=b_{11} b_{22}-b_{12} b_{21}>0$. Then

$$
\begin{aligned}
& f(a, b) f(c, d) \\
= & \sum_{r=0}^{k-1} a^{\frac{r^{2}+r}{2}} b^{\frac{r^{2}-r}{2}} f\left(a^{\frac{b_{11}^{2}+b_{11}}{2}+b_{11} r} b^{\frac{b_{11}^{2}-b_{11}}{2}+b_{11} r} c^{\frac{b_{21}^{2}+b_{21}}{2}} d^{\frac{b_{21}^{2}-b_{21}}{2}}, a^{\frac{b_{11}^{2}-b_{11}}{2}-b_{11} r} b^{\frac{b_{11}^{2}+b_{11}}{2}-b_{11} r} c^{\frac{b_{21}^{2}-b_{21}}{2}} d^{\frac{b_{21}^{2}+b_{21}}{2}}\right) \\
& \times f\left(a^{\frac{b_{12}^{2}+b_{12}}{2}+b_{12} r} b^{\frac{b_{12}^{2}-b_{12}}{2}+b_{12} r} c^{\frac{b_{22}^{2}+b_{22}}{2}} d^{\frac{b_{22}^{2}-b_{22}}{2}}, a^{\frac{b_{12}^{2}-b_{12}}{2}-b_{12} r} b^{\frac{b_{12}^{2}+b_{12}}{2}-b_{12} r} c^{\frac{b_{22}^{2}-b_{22}}{2}} d^{\frac{b_{22}^{2}+b_{22}}{2}}\right) .
\end{aligned}
$$

Proof. If $\operatorname{gcd}\left(b_{21}, b_{11} b_{22}\right)=1$ or $\operatorname{gcd}\left(b_{22}, b_{12} b_{21}\right)=1$, then either $\operatorname{gcd}\left(b_{11}^{*}, k\right)=1$ or $\operatorname{gcd}\left(b_{21}^{*}, k\right)=1$. The conditions of Theorem 1.4 are met.

If $\operatorname{gcd}\left(b_{11}, b_{12} b_{21}\right)=1$ or $\operatorname{gcd}\left(b_{12}, b_{11} b_{22}\right)=1$, then we have the following identity corresponding to $(2.1)$

$$
\begin{aligned}
& f(a, b) f(c, d) \\
= & \sum_{r=0}^{k-1} c^{\frac{r^{2}+r}{2}} d^{\frac{r^{2}-r}{2}} f\left(a^{\frac{b_{11}^{2}+b_{11}}{2}} b^{\frac{b_{11}^{2}-b_{11}}{2}} c^{\frac{b_{21}^{2}+b_{21}}{2}+b_{21} r} d^{\frac{b_{21}^{2}-b_{21}}{2}+b_{21} r}, a^{\frac{b_{11}^{2}-b_{11}}{2}} b^{\frac{b_{11}^{2}+b_{11}}{2}} c^{\frac{b_{21}^{2}-b_{21}}{2}-b_{21} r} d^{\frac{b_{21}^{2}+b_{21}}{2}-b_{21} r}\right) \\
& \times f\left(a^{\frac{b_{12}^{2}+b_{12}}{2}} b^{\frac{b_{12}^{2}-b_{12}}{2}} c^{\frac{b_{22}^{2}+b_{22}}{2}+b_{22} r} d^{\frac{b_{22}^{2}-b_{22}}{2}+b_{22} r}, a^{\frac{b_{12}^{2}-b_{12}}{2}} b^{\frac{b_{12}^{2}+b_{12}}{2}} c^{\frac{b_{22}^{2}-b_{22}}{2}-b_{22} r} d^{\frac{b_{22}^{2}+b_{22}}{2}-b_{22} r}\right) .
\end{aligned}
$$

If $b_{11}$ and $b_{21}, b_{12}$ and $b_{22}$ are of the same parity, then we have the following corollary.

Corollary 2.1. In Theorem [2.1, if we further require that $b_{11}$ and $b_{21}, b_{12}$ and $b_{22}$ are of the same parity, then

$$
f(a, b) f(c, d)+f(-a,-b) f(-c,-d)
$$




$$
\begin{aligned}
& =2 \sum_{r=0}^{\frac{k-2}{2}} a^{2 r^{2}+r} b^{2 r^{2}-r} \\
& f\left(a^{\frac{b_{11}^{2}+b_{11}}{2}+2 b_{11} r} b^{\frac{b_{11}^{2}-b_{11}}{2}+2 b_{11} r} c^{\frac{b_{21}^{2}+b_{21}}{2}} d^{\frac{b_{21}^{2}-b_{21}}{2}}, a^{\frac{b_{11}^{2}-b_{11}}{2}-2 b_{11} r} b^{\frac{b_{11}^{2}+b_{11}}{2}-2 b_{11} r} c^{\frac{b_{21}^{2}-b_{21}}{2}} d^{\frac{b_{21}^{2}+b_{21}}{2}}\right) \\
& \times f\left(a^{\frac{b_{12}^{2}+b_{12}}{2}+2 b_{12} r} b^{\frac{b_{12}^{2}-b_{12}}{2}+2 b_{12} r} c^{\frac{b_{22}^{2}+b_{22}}{2}} d^{\frac{b_{22}^{2}-b_{22}}{2}}, a^{\frac{b_{12}^{2}-b_{12}}{2}-2 b_{12} r} b^{\frac{b_{12}^{2}+b_{12}}{2}-2 b_{12} r} c^{\frac{b_{22}^{2}-b_{22}}{2}} d^{\frac{b_{22}^{2}+b_{22}}{2}}\right) . \\
& f(a, b) f(c, d)-f(-a,-b) f(-c,-d) \\
& =2 \sum_{r=0}^{\frac{k-2}{2}} a^{2 r^{2}+3 r+1} b^{2 r^{2}+r} \\
& f\left(a^{\frac{b_{11}^{2}+3 b_{11}}{2}+2 b_{11} r} b^{\frac{b_{11}^{2}+b_{11}}{2}+2 b_{11} r} c^{\frac{b_{21}^{2}+b_{21}}{2}} d^{\frac{b_{21}^{2}-b_{21}}{2}}, a^{\frac{b_{11}^{2}-3 b_{11}}{2}-2 b_{11} r} b^{\frac{b_{11}^{2}-b_{11}}{2}-2 b_{11} r} c^{\frac{b_{21}^{2}-b_{21}}{2}} d^{\frac{b_{21}^{2}+b_{21}}{2}}\right) \\
& \times f\left(a^{\frac{b_{12}^{2}+3 b_{12}}{2}+2 b_{12} r} b^{\frac{b_{12}^{2}+b_{12}}{2}+2 b_{12} r} c^{\frac{b_{22}^{2}+b_{22}}{2}} d^{\frac{b_{22}^{2}-b_{22}}{2}}, a^{\frac{b_{12}^{2}-3 b_{12}}{2}-2 b_{12} r} b^{\frac{b_{12}^{2}-b_{12}}{2}-2 b_{12} r} c^{\frac{b_{22}^{2}-b_{22}}{2}} d^{\frac{b_{22}^{2}+b_{22}}{2}}\right) .
\end{aligned}
$$

Without lose of generality, it is easy to see that the simplest nontrivial 2 by 2 "generalized orthogonal" matrix is

$$
B=\left(\begin{array}{rr}
1 & 1 \\
-1 & 1
\end{array}\right),
$$

with $\operatorname{det} B=2$. So $k \geqslant 2$ for 2 by 2 matrices throughout the remainder of this paper.

Corollary 2.2. If $|a b|<1$ and $(c d)=(a b)^{k_{1} k_{2}}$, where both $k_{1}$ and $k_{2}$ are positive integers, then

$$
\begin{aligned}
f(a, b) f(c, d)= & \sum_{r=0}^{k_{1}+k_{2}-1} a^{\frac{r^{2}+r}{2}} b^{\frac{r^{2}-r}{2}} f\left(a^{\frac{k_{1}^{2}+k_{1}}{2}+k_{1} r} b^{\frac{k_{1}^{2}-k_{1}}{2}+k_{1} r} d, a^{\frac{k_{1}^{2}-k_{1}}{2}-k_{1} r} b^{\frac{k_{1}^{2}+k_{1}}{2}-k_{1} r} c\right) \\
& \times f\left(a^{\frac{k_{2}^{2}+k_{2}}{2}+k_{2} r} b^{\frac{k_{2}^{2}-k_{2}}{2}+k_{2} r} c, a^{\frac{k_{2}^{2}-k_{2}}{2}-k_{2} r} b^{\frac{k_{2}^{2}+k_{2}}{2}-k_{2} r} d\right) .
\end{aligned}
$$

It is easy too see that (2.6) is symmetric with respect to $a$ and $b, c$ and $d$, and $k_{1}$ and $k_{2}$.

Proof. Choose

$$
B=\left(\begin{array}{rr}
k_{1} & k_{2} \\
-1 & 1
\end{array}\right)
$$

in (2.1).

Many identities involving products of two theta functions are special cases of Corollary 2.2. We give a list of identities here. 


\section{Corollary 2.3.}

$$
\begin{aligned}
\varphi(q) \varphi\left(q^{m}\right) & =\sum_{r=0}^{m} q^{r^{2}} f\left(q^{m+1+2 r}, q^{m+1-2 r}\right) f\left(q^{m^{2}+m+2 m r}, q^{m^{2}+m-2 m r}\right) \\
\varphi(q) \varphi\left(q^{m^{2}}\right) & =\sum_{r=0}^{2 m-1} q^{r^{2}} f^{2}\left(q^{2 m^{2}+2 m r}, q^{2 m^{2}-2 m r}\right) \\
\psi(q) \varphi\left(q^{2 m^{2}}\right) & =\sum_{r=0}^{2 m-1} q^{2 r^{2}-r} f^{2}\left(q^{4 m^{2}-m+4 m r}, q^{4 m^{2}+m-4 m r}\right) \\
2 \psi(q) \psi\left(q^{4 m^{2}}\right) & =\sum_{r=0}^{2 m-1} q^{2 r^{2}-r} f\left(q^{6 m^{2}-m+4 m r}, q^{2 m^{2}+m-4 m r}\right) f\left(q^{2 m^{2}-m+4 m r}, q^{6 m^{2}+m-4 m r}\right) \\
2 \psi(q) \varphi\left(q^{m}\right) & =\sum_{r=0}^{2 m} q^{\frac{r^{2}-r}{2}} f\left(q^{m+r}, q^{m+1-r}\right) f\left(q^{2 m^{2}+m r}, q^{2 m^{2}+2 m-m r}\right) \\
\psi(q) \varphi\left(q^{2 m}\right) & =\sum_{r=0}^{m} q^{2 r^{2}-r} f\left(q^{2 m+1+4 r}, q^{2 m+3-4 r}\right) f\left(q^{2 m^{2}+m+4 m r}, q^{2 m^{2}+3 m-4 m r}\right) \\
2 \varphi(q) \psi\left(q^{2 m}\right) & =\sum_{r=0}^{m} q^{r^{2}} f\left(q^{2 m+1+2 r}, q^{1-2 r}\right) f\left(q^{m^{2}+2 m r}, q^{m^{2}+2 m-2 m r}\right) \\
\psi(q) \psi\left(q^{m}\right) & =\sum_{r=0}^{m} q^{2 r^{2}-r} f\left(q^{3 m+1+4 r}, q^{m+3-4 r}\right) f\left(q^{2 m^{2}+4 m r}, q^{2 m^{2}+4 m-4 m r}\right)
\end{aligned}
$$

If $k_{1} k_{2}$ is a composite number, then the right-hand side of (2.6) is not unique, for it depends on which $k_{1}$ and $k_{2}$ we choose. For example, if we choose $m=4$ in (2.7), we have

$$
\varphi(q) \varphi\left(q^{4}\right)=\varphi\left(q^{5}\right) \phi\left(q^{20}\right)+2 q f\left(q^{3}, q^{7}\right) f\left(q^{12}, q^{28}\right)+2 q^{4} f\left(q, q^{9}\right) f\left(q^{4}, q^{36}\right) .
$$

If we choose $m=2$ in (2.8), we have a different representation of $\varphi(q) \varphi\left(q^{4}\right)$

$$
\varphi(q) \varphi\left(q^{4}\right)=\varphi^{2}\left(q^{8}\right)+2 q \psi^{2}\left(q^{4}\right)+4 q^{4} \psi^{2}\left(q^{16}\right) .
$$

The Göllnitz-Gordon functions are defined by

$$
\begin{aligned}
S(q) & :=\sum_{n=0}^{\infty} \frac{\left(-q ; q^{2}\right)_{n}}{\left(q^{2} ; q^{2}\right)_{n}} q^{n^{2}}=\frac{1}{\left(q ; q^{8}\right)_{\infty}\left(q^{4} ; q^{8}\right)_{\infty}\left(q^{7} ; q^{8}\right)_{\infty}}, \\
T(q) & :=\sum_{n=0}^{\infty} \frac{\left(-q ; q^{2}\right)_{n}}{\left(q^{2} ; q^{2}\right)_{n}} q^{n^{2}+n}=\frac{1}{\left(q^{3} ; q^{8}\right)_{\infty}\left(q^{4} ; q^{8}\right)_{\infty}\left(q^{5} ; q^{8}\right)_{\infty}} .
\end{aligned}
$$

Huang [24, S. L. Chen and S. S. Huang 12 found a list of modular relations for the Göllnitz-Gordon functions which are analogous to Ramanujan's forty identities for the Rogers-Ramanujan functions. Some of their identities can be shown as special cases of our Theorem 2.1 and we give proofs to four of their identities in this paper. 
Corollary 2.4. Let $f_{n}=f\left(-q^{n}\right)$ for nonnegative integer $n$.

$$
\begin{aligned}
S\left(q^{7}\right) T(q)-q^{3} S(q) T\left(q^{7}\right) & =1, \\
S\left(q^{3}\right) S(q)+q^{2} T\left(q^{3}\right) T(q) & =\frac{f_{3} f_{4}}{f_{1} f_{12}}, \\
S(q) S(q)+q T(q) T(q) & =\frac{f_{2}^{6}}{f_{1}^{3} f_{4}^{3}}, \\
S\left(q^{3}\right) T(q)-q S(q) T\left(q^{3}\right) & =\frac{f_{1} f_{12}}{f_{3} f_{4}} .
\end{aligned}
$$

Of Huang's identities, the most beautiful one is (2.15). It is an analogue of Ramanujan's "most beautiful" identity of the forty identities

$$
H(q) G\left(q^{11}\right)-q^{2} G(q) H\left(q^{11}\right)=1,
$$

where

$G(q):=\sum_{n=0}^{\infty} \frac{q^{n^{2}}}{(q ; q)_{n}}=\frac{1}{\left(q ; q^{5}\right)_{\infty}\left(q^{4} ; q^{5}\right)_{\infty}} \quad$ and $\quad H(q):=\sum_{n=0}^{\infty} \frac{q^{n(n+1)}}{(q ; q)_{n}}=\frac{1}{\left(q^{2} ; q^{5}\right)_{\infty}\left(q^{3} ; q^{5}\right)_{\infty}}$.

Proof. If we choose $a=1, b=q, c=1, d=q^{m}, k_{1}=1$ and $k_{2}=m$ in (2.6), then

$$
f(1, q) f\left(1, q^{m}\right)=4 \psi(q) \psi\left(q^{m}\right)=\sum_{r=0}^{m} q^{\frac{r^{2}-r}{2}} f\left(q^{m+r}, q^{1-r}\right) f\left(q^{\frac{m^{2}-m}{2}+m r}, q^{\frac{m^{2}+3 m}{2}-m r}\right) .
$$

We let $m=7$ in (2.19), then apply (1.7) to find that

$$
\begin{aligned}
2 \psi(q) \psi\left(q^{7}\right)= & f\left(q, q^{7}\right) f\left(q^{21}, q^{35}\right)+q^{3} f\left(q^{3}, q^{5}\right) f\left(q^{7}, q^{49}\right) \\
& +q \psi\left(q^{2}\right) \psi\left(q^{14}\right)+\psi\left(q^{8}\right) \varphi\left(q^{28}\right)+q^{6} \varphi\left(q^{4}\right) \psi\left(q^{56}\right) .
\end{aligned}
$$

If we choose $a=-1, b=-q, c=-1, d=-q^{m}, k_{1}=1$ and $k_{2}=m$ in (2.6), by (1.6), $f(-1,-q)=0$. We obtain

$$
\sum_{r=0}^{m}(-1)^{r} q^{\frac{r^{2}-r}{2}} f\left(q^{m+r}, q^{1-r}\right) f\left(q^{\frac{m^{2}-m}{2}+m r}, q^{\frac{m^{2}+3 m}{2}-m r}\right)=0 .
$$

Letting $m=7$ in (2.21), we have

$$
\sum_{i=0}^{7}(-1)^{r} q^{\frac{r^{2}-r}{2}} f\left(q^{7+r}, q^{1-r}\right) f\left(q^{21+7 r}, q^{35-7 r}\right)=0
$$

We can deduce

$$
f\left(q, q^{7}\right) f\left(q^{21}, q^{35}\right)+q^{3} f\left(q^{3}, q^{5}\right) f\left(q^{7}, q^{49}\right)=\psi\left(q^{8}\right) \varphi\left(q^{28}\right)+q^{6} \varphi\left(q^{4}\right) \psi\left(q^{56}\right)+q \psi\left(q^{2}\right) \psi\left(q^{14}\right)
$$

after simplification.

From (2.20) and (2.22), we obtain [2, p. 315, (19.1)]

$$
\psi(q) \psi\left(q^{7}\right)=\psi\left(q^{8}\right) \varphi\left(q^{28}\right)+q^{6} \varphi\left(q^{4}\right) \psi\left(q^{56}\right)+q \psi\left(q^{2}\right) \psi\left(q^{14}\right),
$$


and

$$
\psi(q) \psi\left(q^{7}\right)=f\left(q, q^{7}\right) f\left(q^{21}, q^{35}\right)+q^{3} f\left(q^{3}, q^{5}\right) f\left(q^{7}, q^{49}\right)
$$

Identity (2.23) is equivalent to a modular equation of degree 7 , namely,

$$
(\alpha \beta)^{1 / 8}+\{(1-\alpha)(1-\beta)\}^{1 / 8}=1
$$

where $\beta$ has degree 7 over $\alpha$.

Identity (2.24) is equivalent to (2.15). This proof of (2.24) is simple compared with its proof in [12].

Next we sketch the proofs of

$$
\begin{aligned}
\psi\left(q^{2}\right) \varphi\left(-q^{3}\right) & =f\left(-q^{3},-q^{5}\right) f\left(-q^{9},-q^{15}\right)+q^{2} f\left(-q,-q^{7}\right) f\left(-q^{3},-q^{21}\right), \\
\psi(q) \varphi\left(-q^{2}\right) & =f^{2}\left(-q^{3},-q^{5}\right)+q f^{2}\left(-q,-q^{7}\right), \\
\psi\left(q^{6}\right) \varphi(q) & =f\left(q, q^{7}\right) f\left(q^{9}, q^{15}\right)+q f\left(q^{3}, q^{5}\right) f\left(q^{3}, q^{21}\right),
\end{aligned}
$$

which are equivalent to (2.16), (2.17), and (2.18) respectively. In (2.6), choose $a=1$, $b=q^{2}, c=d=-q^{3}, k_{1}=1$ and $k_{3}=3$. We obtain (2.25) after simplification. In (2.6), choose $a=q, b=q^{3}, c=d=-q^{2}$, and $k_{1}=k_{2}=1$ to obtain (2.26). In (2.6), choose $a=q, b=q, c=1, d=q^{6}, k_{1}=1, k_{2}=3$ and obtain (2.27).

In a manner similar to the derivations of (2.23) and (2.24), we can obtain a list of identities for products of $\psi$ functions after simplification.

\section{Corollary 2.5.}

$$
\begin{aligned}
\psi(q) \psi\left(q^{3}\right) & =\varphi\left(q^{6}\right) \psi\left(q^{4}\right)+q \varphi\left(q^{2}\right) \psi\left(q^{12}\right), \\
\psi(q) \psi\left(q^{5}\right) & =f\left(q, q^{5}\right) f\left(q^{10}, q^{20}\right)+q^{3} \varphi\left(q^{3}\right) \psi\left(q^{30}\right), \\
2 \psi\left(q^{2}\right) \psi\left(q^{3}\right) & =f\left(q^{2}, q^{3}\right) f\left(q^{9}, q^{21}\right)+\psi\left(q^{5}\right) \varphi\left(q^{15}\right)+q^{2} f\left(q, q^{4}\right) f\left(q^{3}, q^{27}\right), \\
\psi\left(q^{3}\right) \psi\left(q^{5}\right) & =f\left(q^{3}, q^{5}\right) f\left(q^{45}, q^{75}\right)+q^{8} f\left(q, q^{7}\right) f\left(q^{15}, q^{105}\right), \\
\psi\left(q^{3}\right) \psi\left(q^{5}\right) & =\psi\left(q^{8}\right) \varphi\left(q^{60}\right)+q^{3} \psi\left(q^{2}\right) \psi\left(q^{30}\right)+q^{14} \varphi\left(q^{4}\right) \psi\left(q^{120}\right), \\
\psi\left(q^{3}\right) \psi\left(q^{7}\right) & =\psi\left(q^{10}\right) \varphi\left(q^{105}\right)+q^{3} f\left(q^{4}, q^{6}\right) f\left(q^{63}, q^{147}\right)+q^{16} f\left(q^{2}, q^{8}\right) f\left(q^{21}, q^{189}\right), \\
\psi\left(q^{3}\right) \psi\left(q^{7}\right) & =f\left(q^{3}, q^{7}\right) f\left(q^{84}, q^{126}\right)+q^{9} f\left(q, q^{9}\right) f\left(q^{42}, q^{168}\right)+q^{25} \varphi\left(q^{5}\right) \psi\left(q^{210}\right), \\
\psi(q) \psi\left(q^{9}\right) & =f\left(q, q^{9}\right) f\left(q^{36}, q^{54}\right)+q^{3} f\left(q^{3}, q^{7}\right) f\left(q^{18}, q^{72}\right)+q^{10} \varphi\left(q^{5}\right) \psi\left(q^{90}\right), \\
\psi(q) \psi\left(q^{9}\right) & =\psi\left(q^{10}\right) \varphi\left(q^{45}\right)+q f\left(q^{2}, q^{8}\right) f\left(q^{27}, q^{63}\right)+q^{6} f\left(q^{4}, q^{6}\right) f\left(q^{9}, q^{81}\right) .
\end{aligned}
$$

If we choose $k_{1}=k_{2}=1$ in (2.6), then we have the following corollary.

Corollary 2.6. For $a b=c d$,

$$
f(a, b) f(c, d)=f(a d, b c) f(a c, b d)+a f\left(c / a, a^{2} b d\right) f\left(d / a, a^{2} b c\right) .
$$

So for $a b=c d$, we have

$$
f(a, b) f(c, d)+f(-a,-b) f(-c,-d)=2 f(a d, b c) f(a c, b d) .
$$

This is (i) of Entry 29 in [2, p. 45]. Similarly we can deduce (ii) of Entry 29, which is

$$
f(a, b) f(c, d)-f(-a,-b) f(-c,-d)=2 a f\left(\frac{b}{c}, \frac{c}{b} a b c d\right) f\left(\frac{b}{d}, \frac{d}{b} a b c d\right)
$$


for $a b=c d$.

We choose $a=b=c=d=q$ in (2.28), then we have

$$
\varphi^{2}(q)=\varphi^{2}\left(q^{2}\right)+4 q \psi^{2}\left(q^{4}\right) .
$$

Replacing $q$ with $q^{2}$ in (2.29), we can obtain

$$
\varphi^{2}(q)=\varphi^{2}\left(q^{4}\right)+4 q \psi^{2}\left(q^{4}\right)+4 q^{2} \psi^{2}\left(q^{8}\right) .
$$

Repeating this process, we have the following formula. For each positive integer $n$,

$$
\varphi^{2}(q)=\varphi^{2}\left(q^{2^{n}}\right)+4 q \psi^{2}\left(q^{4}\right)+\cdots+4 q^{2^{n-1}} \psi^{2}\left(q^{2^{n+1}}\right) .
$$

If we take the square of (2.30), we have the following formula. For each integer $n \geq 2$,

$$
\varphi^{4}(q)=\varphi^{4}\left(q^{2^{n}}\right)+8 q \psi^{4}\left(q^{2}\right)+\sum_{k=1}^{n-1} 24 q^{2^{k}} \psi^{2}\left(q^{2^{k+1}}\right)+16 q^{2^{n}} \psi^{2}\left(q^{2^{n+1}}\right) .
$$

Using (2.28), we can also prove Entry 10 in [3, p. 147], namely

$$
\left[f(a, b)-f\left(a^{3} b, a b^{3}\right)\right]^{2}=f\left(a^{2}, b^{2}\right) \varphi(a b)-f^{2}\left(a^{3} b, a b^{3}\right) .
$$

Proof. From (1.11), we have

$$
f(a, b)-f\left(a^{3} b, a b^{3}\right)=a f\left(b / a, a^{5} b^{3}\right) .
$$

So we only need to show

$$
f\left(a^{2}, b^{2}\right) \varphi(a b)=a^{2} f^{2}\left(b / a, a^{5} b^{3}\right)+f^{2}\left(a^{2} b, a b^{2}\right),
$$

which can be deduced directly from (2.28).

J. A. Ewell's sextuple product identity in [17] is a special case of (2.28).

Corollary 2.7 (Sextuple Product Identity).

$$
\begin{aligned}
& \left(-x y q ; q^{2}\right)_{\infty}\left(-q / x y ; q^{2}\right)_{\infty}\left(-q x / y ; q^{2}\right)_{\infty}\left(-q y / x ; q^{2}\right)_{\infty}\left(q^{2} ; q^{2}\right)_{\infty}^{2} \\
= & \sum_{i=-\infty}^{\infty} q^{2 i^{2}} x^{2 i} \sum_{j=-\infty}^{\infty} q^{2 j^{2}} y^{2 j}+q \sum_{i=-\infty}^{\infty} q^{2 i(i+1)} x^{2 i+1} \sum_{j=-\infty}^{\infty} q^{2 j(j+1)} y^{2 j+1} .
\end{aligned}
$$

Proof. In (2.28), replacing $a$ with $x y q, b$ with $q / x y, c$ with $q x / y$, and $d$ with $q y / x$, and then applying Jacobi's triple product identity, we complete the proof after simplification.

Using (2.28), we can also prove the "three term relation for Weierstrass sigma functions" given in [19, p. 52, Ex. 2.16]. Its equivalent forms appeared before in [29, p. 47] and [33, p. 236].

Corollary 2.8. For nonzero complex numbers $a, b, c, d$,

$$
\begin{aligned}
& b(a d, q / a d, b c, q / b c, d / a, q a / d, c / b, q b / c ; q)_{\infty} \\
+ & c(a b, q / a b, c d, q / c d, b / a, q a / b, d / c, q c / d ; q)_{\infty} \\
+ & d(a c, q / a c, b d, q / b d, c / a, q a / c, b / d, q d / b ; q)_{\infty}=0 .
\end{aligned}
$$


Proof. In (2.28), replacing $a$ with $-a d, b$ with $-q / a d, c$ with $-b c$, and $d$ with $-q / b c$, we have

$$
\langle a d, b c ; q\rangle_{\infty}=\left\langle-a b c d,-a d q / b c ; q^{2}\right\rangle_{\infty}-a d\left\langle-a b c d q,-b c / a d ; q^{2}\right)_{\infty} .
$$

We replace $a$ with $-d / a, b$ with $-q a / d, c$ with $-c / b$, and $d$ with $-q c / d$ in (2.28) to deduce that

$$
\langle d / a, c / b ; q\rangle_{\infty}=\left\langle-c d / a b,-b d q / a c ; q^{2}\right\rangle_{\infty}-d / a\left\langle-a b q / c d,-a c / b d ; q^{2}\right\rangle_{\infty} .
$$

So we have

$$
\begin{aligned}
& b\langle a d, b c, d / a, c / b ; q\rangle_{\infty} \\
= & b\left\langle-a b c d,-a d q / b c,-c d / a b,-b d q / a c ; q^{2}\right\rangle_{\infty}-b d / a\left\langle-a b c d,-a d q / b c,-a b q / c d,-a c / b d ; q^{2}\right\rangle_{\infty} \\
& \left.-a b d\left\langle-a b c d q,-b c / a d,-c d / a b,-b d q / a c ; q^{2}\right)_{\infty}\right)+b d^{2}\left\langle-a b c d q,-b c / a d,-a b q / c d,-a c / b d ; q^{2}\right\rangle_{\infty} .
\end{aligned}
$$

Similarly we have

$$
\begin{aligned}
& c\langle a b, c d, b / a, d / c ; q\rangle_{\infty} \\
= & c\left\langle-a b c d,-a b q / c d,-b d / a c,-a d q / b c ; q^{2}\right\rangle_{\infty}-b c / a\left\langle-a b c d,-a b q / c d,-b d q / a c,-a d / b c ; q^{2}\right\rangle_{\infty} \\
& -a b c\left\langle-a b c d q,-c d / a b,-b d / a c,-a d q / b c ; q^{2}\right\rangle_{\infty}+b^{2} c\left\langle-a b c d q,-c d / a b,-b d q / a c,-a d / b c ; q^{2}\right\rangle_{\infty},
\end{aligned}
$$

and

$$
\begin{aligned}
& d\langle a c, b d, c / a, b / d ; q\rangle_{\infty} \\
= & d\left\langle-a b c d,-a c q / b d,-b c / a d,-c d q / a b ; q^{2}\right\rangle_{\infty}-b\left\langle-a b c d,-a c q / b d,-b c q / a d,-c d / a b ; q^{2}\right\rangle_{\infty} \\
& -b d^{2}\left\langle-a b c d q,-a c / b d,-b c / a d,-c d q / a b ; q^{2}\right\rangle_{\infty}+b^{2} d\left\langle-a b c d q,-a c / b d,-b c q / a d,-c d / a b ; q^{2}\right\rangle_{\infty} .
\end{aligned}
$$

Adding (2.33)-(2.35), we deduce Corollary 2.8 after simplification.

From Corollary 2.2, by choosing

$$
B=\left(\begin{array}{rr}
1 & 2 \\
-1 & 1
\end{array}\right),
$$

we have the following corollary.

Corollary 2.9. For $a b=q$ and $c d=q^{2}$,

$$
\begin{aligned}
f(a, b) f(c, d)= & f(a c, b d) f\left(a^{2} d q, b^{2} c q\right)+a f(a c q, b d / q) f\left(a^{2} d q^{3}, b^{2} c / q\right) \\
& +b f(a c / q, b d q) f\left(a^{2} d / q, b^{2} c q^{3}\right) .
\end{aligned}
$$

We can rewrite Corollary 2.9 in an equivalent form. For $a_{1}, a_{2} \neq 0$,

$$
\begin{aligned}
& \left\langle-a_{1} q ; q^{2}\right\rangle_{\infty}\left\langle-a_{2} q^{2} ; q^{4}\right\rangle_{\infty} \\
= & \left\langle-a_{1} a_{2}{ }^{-1} q^{3} ; q^{6}\right\rangle_{\infty}\left\langle-a_{1}{ }^{2} a_{2} q^{6} ; q^{12}\right\rangle_{\infty}+a_{1} q\left\langle-a_{1} a_{2}{ }^{-1} q^{5} ; q^{6}\right\rangle_{\infty}\left\langle-a_{1}{ }^{2} a_{2} q^{10} ; q^{12}\right\rangle_{\infty} \\
& +a_{1}{ }^{-1} q\left\langle-a_{1} a_{2}{ }^{-1} q ; q^{6}\right\rangle_{\infty}\left\langle-a_{1}{ }^{2} a_{2} q^{2} ; q^{12}\right\rangle_{\infty} .
\end{aligned}
$$

This is Hirschhorn's generalization of the quintuple product identity in [23]. 
In (2.37), we replace $a_{1}$ with $a q, a_{2}$ with $-a^{-2}$, divide both sides by $\left(q^{4} ; q^{4}\right)_{\infty}$, and then replace $q^{2}$ with $q$. We obtain the quintuple product identity in the form of (1.12).

Next we choose

$$
B=\left(\begin{array}{rr}
1 & 2 \\
-2 & 1
\end{array}\right)
$$

in Theorem 2.1 and deduce a generalization of the septuple product identity after simplification.

Corollary 2.10 (Generalized Septuple Product Identity). For $a b=c d=q$,

$$
\begin{aligned}
f(a, b) f(c, d)= & f\left(a c^{2} q, b d^{2} q\right) f\left(a^{2} d q, b^{2} c q\right)+a f\left(a c^{2} q^{2}, b d^{2}\right) f\left(a^{2} d q^{3}, b^{2} c / q\right) \\
& +b f\left(a c^{2}, b d^{2} q^{2}\right) f\left(a^{2} d / q, b^{2} c q^{3}\right)+c f\left(a c^{2} q^{3}, b d^{2} / q\right) f\left(a^{2} d, b^{2} c q^{2}\right) \\
& +d f\left(a c^{2} / q, b d^{2} q^{3}\right) f\left(a^{2} d q^{2}, b^{2} c\right) .
\end{aligned}
$$

We can rewrite (2.39) as follows. For $a_{1}, a_{2} \neq 0$,

$$
\begin{aligned}
& \left\langle-a_{1} ; q^{2}\right\rangle_{\infty}\left\langle-a_{2} ; q^{2}\right\rangle_{\infty} \\
= & \left\langle-a_{1} a_{2}{ }^{2} q^{2} ; q^{10}\right\rangle_{\infty}\left\langle-a_{1}{ }^{2} a_{2}{ }^{-1} q^{4} ; q^{10}\right\rangle_{\infty}+a_{1}\left\langle-a_{1} a_{2}{ }^{2} q^{4} ; q^{10}\right\rangle_{\infty}\left\langle-a_{1}{ }^{2} a_{2}{ }^{-1} q^{8} ; q^{10}\right\rangle_{\infty} \\
& +a_{2}\left\langle-a_{1} a_{2}{ }^{2} q^{6} ; q^{10}\right\rangle_{\infty}\left\langle-a_{1}{ }^{2} a_{2}{ }^{-1} q^{2} ; q^{10}\right\rangle_{\infty}+a_{1} a_{2}\left\langle-a_{1} a_{2}{ }^{2} q^{8} ; q^{10}\right\rangle_{\infty}\left\langle-a_{1}{ }^{2} a_{2}{ }^{-1} q^{6} ; q^{10}\right\rangle_{\infty} \\
& +a_{1} a_{2}{ }^{-1} q^{2}\left\langle-a_{1} a_{2}{ }^{2} ; q^{10}\right\rangle_{\infty}\left\langle-a_{1}{ }^{2} a_{2}{ }^{-1} q^{10} ; q^{10}\right\rangle_{\infty} .
\end{aligned}
$$

Replacing $a_{1}$ with $-a$ and $a_{2}$ with $-a^{2}$ in (2.39), we have the septuple product identity in the form of (1.13).

Identity (2.38) leads to two of Ramanujan's modular identities which are very important in the study of the modular equations of degree 5. We cite the identities in [2, p. 262, Entry 10 (iv) and (v)].

\section{Corollary 2.11.}

$$
\begin{aligned}
\varphi^{2}(q)-\varphi^{2}\left(q^{5}\right) & =4 q f\left(q, q^{9}\right) f\left(q^{3}, q^{7}\right), \\
\psi^{2}(q)-q \psi^{2}\left(q^{5}\right) & =f\left(q, q^{4}\right) f\left(q^{2}, q^{3}\right) .
\end{aligned}
$$

Proof. For (2.40), by choosing $a=b=c=d=q$ in (2.38), we can deduce (2.40) after simplification.

For (2.41), by choosing $a=c=1, b=d=q$ in (2.38), we have $f(1, q)-q f\left(1, q^{5}\right)=$ $4 f\left(q, q^{4}\right) f\left(q^{2}, q^{3}\right)$, which is equivalent to (2.41).

On page 207 of his lost notebook, Ramanujan listed three identities

$$
\begin{aligned}
P-Q & =1+\frac{f\left(-q^{1 / 5},-\lambda q^{2 / 5}\right)}{q^{1 / 5} f\left(-\lambda^{10} q^{5},-\lambda^{15} q^{10}\right)}, \\
P Q & =1-\frac{f\left(-\lambda,-\lambda^{4} q^{3}\right) f\left(-\lambda^{2} q,-\lambda^{3} q^{2}\right)}{f^{2}\left(-\lambda^{10} q^{5},-\lambda^{15} q^{10}\right)}, \\
P^{5}-Q^{5} & =1+5 P Q+5 P^{2} Q^{2}+\frac{f\left(-q,-\lambda^{5} q^{2}\right) f^{5}\left(-\lambda^{2} q,-\lambda^{3} q^{2}\right)}{q f^{6}\left(-\lambda^{10} q^{5},-\lambda^{15} q^{10}\right)}
\end{aligned}
$$


without specifying $P$ and $Q$. S. H. Son [32] first determined the functions $P$ and $Q$. His proof of (2.43) is equivalent to the proof of the identity

$$
\begin{aligned}
S:= & f\left(-\lambda,-\lambda^{4} q^{3}\right) f\left(-\lambda^{2} q,-\lambda^{3} q^{2}\right) \\
= & f^{2}\left(-\lambda^{10} q^{5},-\lambda^{15} q^{10}\right)-\lambda f\left(-\lambda^{5} q^{4},-\lambda^{20} q^{11}\right) f\left(-\lambda^{10} q^{7},-\lambda^{15} q^{8}\right) \\
& -\lambda^{2} q f\left(-\lambda^{5} q^{4},-\lambda^{20} q^{11}\right) f\left(-\lambda^{5} q^{2},-\lambda^{20} q^{13}\right)+\lambda^{3} q f\left(-q,-\lambda^{25} q^{14}\right) f\left(-\lambda^{10} q^{7},-\lambda^{15} q^{8}\right) \\
& +\lambda^{4} q^{2} f\left(-q,-\lambda^{25} q^{14}\right) f\left(-\lambda^{5} q^{2},-\lambda^{20} q^{13}\right) .
\end{aligned}
$$

Observing that $-\lambda \cdot-\lambda^{4} q^{3}=-\lambda^{2} q \cdot-\lambda^{3} q^{2}=\lambda^{5} q^{3}$, and the right-hand side of (2.45) is the sum of five parts. We choose $a=-\lambda, b=-\lambda^{4} q^{3}, c=-\lambda^{2} q$, and $d=-\lambda^{3} q^{2}$ in (2.38). Then (2.45) follows immediately.

Define the septic Rogers-Ramanujan functions

$$
\begin{aligned}
& A(q):=\sum_{n=0}^{\infty} \frac{q^{2 n^{2}}}{\left(q^{2} ; q^{2}\right)_{n}(-q ; q)_{2 n}}=\frac{\left(q^{7} ; q^{7}\right)_{\infty}\left(q^{3} ; q^{7}\right)_{\infty}\left(q^{4} ; q^{7}\right)_{\infty}}{\left(q^{2} ; q^{2}\right)_{\infty}}=\frac{f\left(-q^{3},-q^{4}\right)}{f\left(-q^{2}\right)}, \\
& B(q):=\sum_{n=0}^{\infty} \frac{q^{2 n(n+1)}}{\left(q^{2} ; q^{2}\right)_{n}(-q ; q)_{2 n}}=\frac{\left(q^{7} ; q^{7}\right)_{\infty}\left(q^{2} ; q^{7}\right)_{\infty}\left(q^{5} ; q^{7}\right)_{\infty}}{\left(q^{2} ; q^{2}\right)_{\infty}}=\frac{f\left(-q^{2},-q^{5}\right)}{f\left(-q^{2}\right)} \\
& C(q):=\sum_{n=0}^{\infty} \frac{q^{2 n^{2}}}{\left(q^{2} ; q^{2}\right)_{n}(-q ; q)_{2 n}}=\frac{\left(q^{7} ; q^{7}\right)_{\infty}\left(q ; q^{7}\right)_{\infty}\left(q^{6} ; q^{7}\right)_{\infty}}{\left(q^{2} ; q^{2}\right)_{\infty}}=\frac{f\left(-q,-q^{6}\right)}{f\left(-q^{2}\right)}
\end{aligned}
$$

where the equalities are from L. J. Slater [31]. H. Hahn [20] obtained many identities for the septic Rogers-Ramanujan functions. Identities (3.1)-(3.13) of [20] are special cases of Theorem [1.4. Next we prove (3.1) of [20] as an example and omit the proofs of the others.

$$
A_{1} B_{3}-q B_{1} C_{3}-C_{1} A_{3}=0
$$

Here

$$
A_{n}:=A\left(q^{n}\right), \quad B_{n}:=B\left(q^{n}\right), \quad C_{n}:=C\left(q^{n}\right)
$$

for positive $n$.

Proof. After simplification, we can write (2.46) as

$$
f\left(-q^{3},-q^{4}\right) f\left(-q^{6},-q^{15}\right)-q f\left(-q^{2},-q^{5}\right) f\left(-q^{3},-q^{18}\right)-f\left(-q,-q^{6}\right) f\left(-q^{9},-q^{12}\right)=0 .
$$

Since $-q^{3} \cdot-q^{4}=-q^{2} \cdot-q^{5}=-q \cdot-q^{6}=q^{7},-q^{6} \cdot-q^{15}=-q^{3} \cdot-q^{18}=-q^{9} \cdot-q^{12}=q^{21}$, by Theorem [2.1, we need to solve the system of equations

$$
\left\{\begin{array}{l}
l_{1} b_{11}^{2}+l_{2} b_{21}^{2}=7 \\
l_{1} b_{12}^{2}+l_{2} b_{22}^{2}=21
\end{array}\right.
$$

with $l_{i}$ and $b_{i j}$ as unknown integers. We can find that $l_{1}=1, l_{2}=3$. The solutions $b_{i j}$ 's to equation (2.48) differ only by signs, and they essentially generate the same identity. So we can choose

$$
B=\left(\begin{array}{rr}
2 & 3 \\
-1 & 2
\end{array}\right) \text {. }
$$


We omit the details here. Note that $k=\operatorname{det} B=7$. As before, adding up the contribution of each member of the ECS corresponding to $B$, we can deduce that the left-hand side of (2.47) is $\frac{1}{2} f(-1,-q) f\left(-1,-q^{3}\right)$. Since $f(-1, a)=0$ for any $|a|<1$, (2.47) holds.

Identity (2.46) is not a special case of Corollary 2.2. The reason is if we use Corollary 2.2, then based on $l_{1}=1$ and $l_{2}=3$, the simplest matrix

$$
B=\left(\begin{array}{rr}
1 & 3 \\
-1 & 1
\end{array}\right)
$$

would arise, but this $B$ cannot be used to deduce (2.46).

Schröter's formula first appeared in H. Schröter's dissertation in 1854. It is very useful in proving many of Ramanujan's modular equations. Next we show that Theorem 2.1 is also a generalization of Schröter's formula and derive several generalized forms of Schröter's formula. We use the notation of Schröter's formula in [7].

Let a general theta function be defined as: $T(x, q)=\sum_{n=-\infty}^{\infty} x^{n} q^{n^{2}}$, where $x \neq 0$, $|q|<1$. It is obvious that $T(x, q)=T(1 / x, q)$ from this definition.

Theorem 2.2 (Schröter's Formula). For positive integers $a, b$,

$$
T\left(x, q^{a}\right) T\left(y, q^{b}\right)=\sum_{n=0}^{a+b-1} y^{n} q^{b n^{2}} T\left(x y q^{2 b n} ; q^{a+b}\right) T\left(x^{-b} y^{a} q^{2 a b n}, q^{a b^{2}+a^{2} b}\right) .
$$

Since $T(x, q)=f(x q, q / x)$, it is easy to see that Theorem 2.1 is a generalization of Schröter's formula. If we use the notations of [7], we can rewrite Theorem 2.1] as below.

Theorem 2.3 (Generalized Schröter's Formula). For positive integers $a, b$, we can find an $2 \times 2$ invertible integer matrix $B$ such that $a b_{11} b_{12}+b b_{21} b_{22}=0, \operatorname{gcd}\left(b_{21}, b_{11} b_{22}\right)=1$ or $\operatorname{gcd}\left(b_{22}, b_{12} b_{21}\right)=1$. Let $k=\operatorname{det} B>0$. Then

$$
T\left(x, q^{a}\right) T\left(y, q^{b}\right)=\sum_{n=0}^{k-1} y^{n} q^{b n^{2}} T\left(x^{b_{11}} y^{b_{21}} q^{2 b b_{21} n} ; q^{a b_{11}{ }^{2}+b b_{21}{ }^{2}}\right) T\left(x^{b_{12}} y^{b_{22}} q^{2 b b_{22} n} ; q^{a b_{12}{ }^{2}+b b_{22}{ }^{2}}\right) .
$$

Corollary 2.12. For positive integers $a, b, k_{i}(i=1,2)$, we have

$T\left(x, q^{a}\right) T\left(y, q^{b}\right)=\sum_{n=0}^{k_{2} a+k_{1}^{2} k_{2} b-1} y^{n} q^{b n^{2}} T\left(x y^{k_{1}} q^{2 k_{1} b n} ; q^{a+b k_{1}{ }^{2}}\right) T\left(x^{-k_{1} k_{2} b} y^{k_{2} a} q^{2 k_{2} a b n}, q^{k_{1}{ }^{2} k_{2}{ }^{2} a b^{2}+k_{2}{ }^{2} a^{2} b}\right)$.

Proof. Choose

$$
B=\left(\begin{array}{rr}
1 & -k_{1} k_{2} b \\
k_{1} & k_{2} a
\end{array}\right)
$$


If we choose $k_{1}=k_{2}=1$ in (2.51), we obtain Schröter's formula, which corresponds to

$$
B=\left(\begin{array}{rr}
1 & -b \\
1 & a
\end{array}\right) .
$$

We show that S. Kongsiriwong and Z.-G. Liu's Theorems 6-9 in [26] are special cases of Corollary 2.12.

Corollary 2.13 (Theorems $6-9$ in [26]). Let $z$ and $q$ be complex numbers with $z \neq 0$ and $|q|<1$. Then, for any positive integer $k$,

$$
\begin{aligned}
& \sum_{j=0}^{[(k-1) / 2]}(-1)^{j} q^{j^{2}} \sum_{m=-\infty}^{\infty}(-1)^{(k-1) m} q^{\left(k^{2}+k\right) m^{2}-(2 j+1) k m} \\
& \times \sum_{n=-\infty}^{\infty} q^{(k+1) n^{2}} z^{(k+1) n}\left(q^{(2 j-k) n} z^{j}-q^{(j-2 k) n} z^{k-j}\right) \\
& =\left(q^{2} ; q^{2}\right)_{\infty}\left(z q ; q^{2}\right)_{\infty}\left(z^{-1} q ; q^{2}\right)_{\infty}\left(q^{2 k} ; q^{2 k}\right)_{\infty}\left(z^{k} ; q^{2 k}\right)_{\infty}\left(z^{-k} q^{2 k} ; q^{2 k}\right)_{\infty}, \\
& \frac{1-(-1)^{k}}{2}(-1)^{(k+1) / 2} q^{(k+1)^{2} / 4} \sum_{m=-\infty}^{\infty}(-1)^{(k-1) m} q^{\left(k^{2}+k\right) m^{2}+\left(k^{2}+k\right) m} \\
& \times \sum_{n=-\infty}^{\infty} q^{(k+1) n^{2}-(k+1) n} z^{(k+1) n-(k+1) / 2} \\
& +\sum_{m=-\infty}^{\infty}(-1)^{(k-1) m} q^{\left(k^{2}+k\right) m^{2}} \sum_{n=-\infty}^{\infty} q^{(k+1) n^{2}} z^{(k+1) n} \\
& +\sum_{j=1}^{[k / 2]}(-1)^{j} q^{j^{2}} \sum_{m=-\infty}^{\infty}(-1)^{(k-1) m} q^{\left(k^{2}+k\right) m^{2}-2 j k m} \sum_{n=-\infty}^{\infty} q^{(k+1) n^{2}} z^{(k+1) n}\left(q^{2 j n} z^{j}-q^{-2 j n} z^{-j}\right) \\
& =\left(q^{2} ; q^{2}\right)_{\infty}\left(z q ; q^{2}\right)_{\infty}\left(z^{-1} q ; q^{2}\right)_{\infty}\left(q^{2 k} ; q^{2 k}\right)_{\infty}\left(z^{k} q^{k} ; q^{2 k}\right)_{\infty}\left(z^{-k} q^{k} ; q^{2 k}\right)_{\infty}, \\
& \sum_{j=-k+1}^{k}(-1)^{j} q^{j^{2}-j} \sum_{m=-\infty}^{\infty}(-1)^{m} q^{\left(4 k^{2}+k\right) m^{2}+(1-4 j) k m} \\
& \times \sum_{n=-\infty}^{\infty}(-1)^{n} q^{(4 k+1) n^{2}} z^{(4 k+1) n}\left(q^{(2 j-2 k-1) n} z^{j}-q^{(2 k+1-2 j) n} z^{2 k+1-j}\right) \\
& =\left(q^{2} ; q^{2}\right)_{\infty}\left(z ; q^{2}\right)_{\infty}\left(z^{-1} q^{2} ; q^{2}\right)_{\infty}\left(q^{2 k} ; q^{2 k}\right)_{\infty}\left(z^{2 k} ; q^{2 k}\right)_{\infty}\left(z^{-2 k} q^{2 k} ; q^{2 k}\right)_{\infty}, \\
& \sum_{m=-\infty}^{\infty}(-1)^{m} q^{\left(4 k^{2}+k\right) m^{2}} \sum_{n=-\infty}^{\infty}(-1)^{n} q^{(4 k+1) n^{2}} z^{(4 k+1) n} \\
& +\sum_{j=1}^{2 k}(-1)^{j} q^{j^{2}} \sum_{m=-\infty}^{\infty}(-1)^{m} q^{\left(4 k^{2}+k\right) m^{2}-4 j k m} \sum_{n=-\infty}^{\infty}(-1)^{n} q^{(4 k+1) n^{2}} z^{(4 k+1) n}\left(q^{2 j n} z^{j}+q^{-2 j n} z^{-j}\right) \\
& =\left(q^{2} ; q^{2}\right)_{\infty}\left(z q ; q^{2}\right)_{\infty}\left(z^{-1} q ; q^{2}\right)_{\infty}\left(q^{2 k} ; q^{2 k}\right)_{\infty}\left(z^{2 k} q^{k} ; q^{2 k}\right)_{\infty}\left(z^{-2 k} q^{k} ; q^{2 k}\right)_{\infty} \text {. }
\end{aligned}
$$


Proof. For (2.52) and (2.53), choose the matrix

$$
B=\left(\begin{array}{rr}
1 & -k \\
1 & 1
\end{array}\right)
$$

this corresponds to the case $a=1$ and $b=k$ in Schröter's formula (2.49).

For (2.54) and (2.55), choose

$$
B=\left(\begin{array}{rr}
1 & -2 k \\
2 & 1
\end{array}\right)
$$

this corresponds to $a=1, b=k, k_{1}=2$, and $k_{2}=1$ in (2.51). We omit the details.

If we choose $k_{1}=1$ and $k_{2}=k$ in (2.51), we deduce the following corollary.

Corollary 2.14. For positive integers $a, b, k$,

$$
T\left(x, q^{a}\right) T\left(y, q^{b}\right)=\sum_{n=0}^{k a+k b-1} y^{n} q^{b n^{2}} T\left(x y q^{2 b n} ; q^{a+b}\right) T\left(x^{-k b} y^{k a} q^{2 k a b n}, q^{k^{2} a b(a+b)}\right) .
$$

We can obtain (2.56) directly from Schröter's formula (2.49) by replacing $q$ with $q^{1 / k}$, $a$ with $k a$, and $b$ with $k b$.

If we choose $k_{1}=k$ and $k_{2}=1$ in (2.51), then we arrive at the next corollary.

Corollary 2.15. For positive integers $a, b, k$,

$$
T\left(x, q^{a}\right) T\left(y, q^{b}\right)=\sum_{n=0}^{a+k^{2} b-1} y^{n} q^{b n^{2}} T\left(x y^{k} q^{2 b k n}, q^{a+b k^{2}}\right) T\left(x^{-k b} y^{a} q^{2 a b n}, q^{k^{2} a b^{2}+a^{2} b}\right) .
$$

If we choose $k=2, a=3, b=1, x=-q^{-3}, y=-q^{-1}$ in (2.57), and then replace $q^{2}$ with $q$, we can obtain Hahn's identity (2.46).

Assuming that $k_{2} \mid a$, we can also derive a variation of (2.51) by replacing $k_{2}$ with $1 / k_{2}$.

Corollary 2.16. For positive integers $a, b, k_{i}(i=1,2), k_{2} \mid k_{1} b$, and $k_{2} \mid a$, we have

$$
\begin{aligned}
& T\left(x, q^{a}\right) T\left(y, q^{b}\right) \\
= & \sum_{n=0}^{a / k_{2}+k_{1}^{2} b / k_{2}-1} y^{n} q^{b n^{2}} T\left(x y^{k_{1}} q^{2 k_{1} b n} ; q^{a+b k_{1}{ }^{2}}\right) T\left(x^{-k_{1} b / k_{2}} y^{a / k_{2}} q^{2 a b n / k_{2}}, q^{a b^{2} k_{1}{ }^{2} / k_{2}{ }^{2}+a^{2} b / k_{2}{ }^{2}}\right) .
\end{aligned}
$$

We obtain the next corollary if we choose the transformation matrix

$$
B=\left(\begin{array}{rr}
1 & -k_{1} k_{2} b \\
k_{1} a & k_{2}
\end{array}\right)
$$

Corollary 2.17. For positive integers $a, b, k_{i}(i=1,2)$, we have

$$
\begin{aligned}
& T\left(x, q^{a}\right) T\left(y, q^{b}\right) \\
= & \sum_{n=0}^{k_{2}+k_{1}^{2} k_{2} a b-1} y^{n} q^{b n^{2}} T\left(x y^{k_{1} a} q^{2 k_{1} a b n} ; q^{a+b k_{1}{ }^{2} a^{2}}\right) T\left(x^{-k_{1} k_{2} b} y^{k_{2}} q^{2 k_{2} a b n}, q^{k_{1}{ }^{2} k_{2}{ }^{2} a b^{2}+k_{2}{ }^{2} b}\right) .
\end{aligned}
$$


We can obtain Corollary 2.17 by replacing $k_{1}$ with $k_{1} a$ and $k_{2} a$ with $k_{2}$ in Corollary 2.12 ,

In [14], W. Chu and Q. Yan obtained a general formula with many known identities as special cases. The main Theorem in Chu and Yan's paper is the following result.

Corollary 2.18. Let $\alpha, \beta, \gamma$ be three natural integers with $\operatorname{gcd}(\alpha, \gamma)=1$ and $\lambda=$ $1+\alpha \beta^{2} \gamma$. For two indeterminates $x$ and $y$ with $x \neq 0$ and $y \neq 0$, there holds the algebraic identity

$$
\begin{aligned}
\left\langle x ; q^{\alpha}\right\rangle_{\infty}\left\langle x^{\beta \gamma} y ; q^{\gamma}\right\rangle_{\infty}= & \sum_{l=0}^{\alpha \beta^{2} \gamma}(-1)^{l} q^{\left(\begin{array}{c}
l \\
2
\end{array}\right) \alpha} x^{l}\left\langle(-1)^{\alpha \beta} x^{\lambda} y^{\alpha \beta} q^{\left(\begin{array}{c}
\alpha \beta \\
2
\end{array}\right) \gamma+l \alpha} ; q^{\lambda \alpha}\right\rangle \\
& \times\left\langle(-1)^{\beta \gamma} y q^{\left(\begin{array}{c}
\beta \gamma+1 \\
2
\end{array}\right) \gamma-l \alpha \beta \gamma} ; q^{\lambda \gamma}\right\rangle .
\end{aligned}
$$

This corollary is a special case of both (2.58) and (2.59). If we choose $k_{1}=\alpha \beta$, $k_{2}=\alpha, a=\alpha$, and $b=\gamma$, replace $x$ with $-q^{-\alpha / 2} x, y$ with $-x^{\beta \gamma} y q^{-\gamma / 2}, q$ with $q^{1 / 2}$ in (2.58), and finally change the notation $n$ to $l$, we obtain (2.60). Or if we choose $k_{1}=\beta, k_{2}=1, a=\alpha$, and $b=\gamma$, replace $x$ with $-q^{-\alpha / 2} x, y$ with $-x^{\beta \gamma} y q^{-\gamma / 2}, q$ with $q^{1 / 2}$ in (2.59), and finally change the notation $n$ to $l$ to obtain (2.60). Identity (2.60) is corresponding to

$$
B=\left(\begin{array}{rr}
\beta \gamma & -1 \\
1 & \alpha \beta
\end{array}\right)
$$

If we choose $k_{1}=k_{2}=k$ in (2.58), we can deduce the next corollary.

Corollary 2.19. For positive integers $a, b, k$, such that $k \mid a$,

$$
T\left(x, q^{a}\right) T\left(y, q^{b}\right)=\sum_{n=0}^{\frac{a}{k}+b k-1} y^{n} q^{b n^{2}} T\left(x y^{k} q^{2 b k n}, q^{a+b k^{2}}\right) T\left(x^{-b} y^{\frac{a}{k}} q^{\frac{2 a b}{k} n}, q^{a b^{2}+\frac{a^{2} b}{k^{2}}}\right) .
$$

The Blecksmith-Brillhart-Gerst theorem in [6, Theorem 2], which is a nice generalization of Schröter's formula, can be shown to be a special case of (2.61). We cite the theorem in [2, p. 73], which uses Ramanujan's notation.

Corollary 2.20. Define $f_{0}(a, b)=f(a, b)$ and $f_{1}(a, b)=f(-a,-b)$. Let $a, b, c$ and $d$ denote complex numbers such that $|a b|,|c d|<1$. Suppose that there exist positive integers $\alpha, \beta$, and $m$ such that

$$
(a b)^{\beta}=(c d)^{\alpha(m-\alpha \beta)} .
$$

Let $\varepsilon_{1}, \varepsilon_{2} \in\{0,1\}$. Then

$$
\begin{aligned}
& f_{\varepsilon_{1}}(a, b) f_{\varepsilon_{2}}(c, d) \\
= & \sum_{r \in R}(-1)^{\varepsilon_{2} r} c^{r(r+1) / 2} d^{r(r-1) / 2} f_{\delta_{1}}\left(\frac{a(c d)^{\alpha(\alpha+1-2 n) / 2}}{c^{\alpha}}, \frac{b(c d)^{\alpha(\alpha+1+2 n) / 2}}{d^{\alpha}}\right) \\
& \times f_{\delta_{2}}\left(\frac{(a / b)^{\beta / 2}(c d)^{(m-\alpha \beta)(m+1+2 n) / 2}}{d^{m-\alpha \beta}}, \frac{(b / a)^{\beta / 2}(c d)^{(m-\alpha \beta)(m+1-2 n) / 2}}{c^{m-\alpha \beta}}\right),
\end{aligned}
$$


where $R$ is a complete residue system $(\bmod m)$,

$$
\delta_{1}= \begin{cases}0, & \text { if } \varepsilon_{1}+\alpha \varepsilon_{2} \text { is even, } \\ 1, & \text { if } \varepsilon_{1}+\alpha \varepsilon_{2} \text { is odd }\end{cases}
$$

and

$$
\delta_{2}= \begin{cases}0, & \text { if } \varepsilon_{1} \beta+\varepsilon_{2}(m-\alpha \beta) \text { is even, } \\ 1, & \text { if } \varepsilon_{1} \beta+\varepsilon_{2}(m-\alpha \beta) \text { is odd. }\end{cases}
$$

Proof. Replacing $x$ with $(-1)^{\varepsilon_{1}} x$, and $y$ with $(-1)^{\varepsilon_{2}} y$ in (2.61), we have

$$
\begin{aligned}
T\left((-1)^{\varepsilon_{1}} x, q^{a}\right) T\left((-1)^{\varepsilon_{2}} y, q^{b}\right)= & \sum_{n=0}^{\frac{a}{k}+b k-1}(-1)^{\varepsilon_{2} n} y^{n} q^{b n^{2}} T\left((-1)^{\varepsilon_{1}+k \varepsilon_{2}} x y^{k} q^{2 b k n}, q^{a+b k^{2}}\right) \\
& \times T\left((-1)^{b \varepsilon_{1}+\frac{a}{k} \varepsilon_{2}} x^{-b} y^{\frac{a}{k}} q^{\frac{2 a b}{k} n}, q^{a b^{2}+\frac{a^{2} b}{k^{2}}}\right) .
\end{aligned}
$$

We replace $x$ with $(b / a)^{1 / 2}, q^{a}$ with $(a b)^{1 / 2}, y$ with $(c / d)^{1 / 2}, q^{b}$ with $(c d)^{1 / 2}$, and $k$ with $\alpha$ in (2.62), where $a, b, c, d$ are complex numbers such that $|a b|<1,|c d|<1$ and $\alpha, \beta, m$ are positive integers. We choose $a=\alpha(m-\alpha \beta)$ and $b=\beta$ such that $(a b)^{\beta}=(c d)^{\alpha(m-\alpha \beta)}$. By (2.62),

$$
\begin{aligned}
& T\left((-1)^{\varepsilon_{1}}(b / a)^{1 / 2},(a b)^{1 / 2}\right) T\left((-1)^{\varepsilon_{2}}(c / d)^{1 / 2},(c d)^{1 / 2}\right) \\
= & \sum_{n=0}^{m-1}(-1)^{\varepsilon_{2}}(c / d)^{n / 2}(c d)^{n^{2} / 2} T\left((-1)^{\varepsilon_{1}+\alpha \varepsilon_{2}}(b / a)^{1 / 2}(c / d)^{\alpha / 2}(c d)^{\alpha n} ;(a b)^{1 / 2}(c d)^{\alpha^{2} / 2}\right) \\
& \times T\left((-1)^{\beta \varepsilon_{1}+(m-\alpha \beta) \varepsilon_{2}}(a / b)^{\beta / 2}(c / d)^{(m-\alpha \beta) / 2}(c d)^{m-\alpha \beta n},(a b)^{\beta^{2} / 2}(c d)^{(m-\alpha \beta)^{2} / 2}\right) .
\end{aligned}
$$

After simplification, we have

$$
\begin{aligned}
& f\left((-1)^{\varepsilon_{1}} a,(-1)^{\varepsilon_{1}} b\right) f\left((-1)^{\varepsilon_{2}} c,(-1)^{\varepsilon_{2}} d\right) \\
= & \sum_{n=0}^{m-1}(-1)^{\varepsilon_{2}} c^{n(n+1) / 2} d^{n(n-1) / 2} f\left((-1)^{\varepsilon_{1}+\alpha \varepsilon_{2}} \frac{b(c d)^{\alpha(\alpha+1+2 n) / 2}}{d^{\alpha}},(-1)^{\varepsilon_{1}+\alpha \varepsilon_{2}} \frac{a(c d)^{\alpha(\alpha+1-2 n) / 2}}{c^{\alpha}}\right) \\
& \times f\left((-1)^{\beta \varepsilon_{1}+(m-\alpha \beta) \varepsilon_{2}} \frac{(a / b)^{\beta / 2}(c d)^{(m-\alpha \beta)(m+1+2 n) / 2}}{d^{m-\alpha \beta}},\right. \\
& \left.(-1)^{\beta \varepsilon_{1}+(m-\alpha \beta) \varepsilon_{2}} \frac{(b / a)^{\beta / 2}(c d)^{(m-\alpha \beta)(m+1-2 n) / 2}}{c^{m-\alpha \beta}}\right) .
\end{aligned}
$$

Here the corresponding matrix is

$$
B=\left(\begin{array}{rr}
1 & -\beta \\
\alpha & m-\alpha \beta
\end{array}\right)
$$

If we choose $k_{1}=1, k_{2}=k$ in (2.58), we can deduce the following corollary. 
Corollary 2.21. For positive integers $a, b, k$, such that $k \mid a$ and $k \mid b$,

$$
T\left(x, q^{a}\right) T\left(y, q^{b}\right)=\sum_{n=0}^{\frac{a+b}{k}-1} y^{n} q^{b n^{2}} T\left(x y q^{2 b n}, q^{a+b}\right) T\left(x^{-\frac{b}{k}} y^{\frac{a}{k}} q^{\frac{2 a b}{k} n}, q^{\frac{a b(a+b)}{k^{2}}}\right) .
$$

We can obtain Corollary 2.21 by replacing $q$ with $q^{k}$, $a$ with $a / k$, and $b$ with $b / k$ in Schröter's formula (2.49).

For each of the above results related to Schröter's formula, we can switch the positions of $x$ and $y$, and $a$ and $b$ at the same time since their positions are symmetric. We can also replace $x$ with $1 / x$ and $y$ with $1 / y$ on the right-hand sides of all such formulas.

The general approach we developed previously can also be applied to some infinite sums that are not theta functions. The idea is for any infinite sum, we want all the coefficients of $y_{i} y_{j}(i \neq j)$ terms to be zero after changing the variables from $x_{1}, \ldots, x_{n}$ to $y_{1}, \ldots, y_{n}$. For example, we can consider the following infinite sum

$$
\sum_{x_{1}, x_{2}=-\infty}^{\infty} q^{x_{1}^{2}+x_{1} x_{2}+x_{2}^{2}}
$$

We change the variables from $x_{1}, x_{2}$ to $y_{1}, y_{2}$ by the integral matrix exact covering system

$$
\left\{\left(\begin{array}{l}
x_{1} \\
x_{2}
\end{array}\right)=\left(\begin{array}{rr}
1 & 1 \\
-1 & 1
\end{array}\right)\left(\begin{array}{l}
y_{1} \\
y_{2}
\end{array}\right), \quad\left(\begin{array}{l}
x_{1} \\
x_{2}
\end{array}\right)=\left(\begin{array}{rr}
1 & 1 \\
-1 & 1
\end{array}\right)\left(\begin{array}{l}
y_{1} \\
y_{2}
\end{array}\right)+\left(\begin{array}{l}
1 \\
0
\end{array}\right)\right\} .
$$

So we can write the infinite sum (2.64) as the linear combination of two parts

$$
\begin{aligned}
& \sum_{x_{1}, x_{2}=-\infty}^{\infty} q^{x_{1}{ }^{2}+x_{1} x_{2}+x_{2}{ }^{2}} \\
= & \sum_{y_{1}, y_{2}=-\infty}^{\infty} q^{\left(y_{1}+y_{2}\right)^{2}+\left(y_{1}+y_{2}\right)\left(-y_{1}+y_{2}\right)+\left(-y_{1}+y_{2}\right)^{2}}+\sum_{y_{1}, y_{2}=-\infty}^{\infty} q^{\left(y_{1}+y_{2}+1\right)^{2}+\left(y_{1}+y_{2}+1\right)\left(-y_{1}+y_{2}\right)+\left(-y_{1}+y_{2}\right)^{2}} \\
= & \sum_{y_{1}, y_{2}=-\infty}^{\infty} q^{y_{1}{ }^{2}+3 y_{2}{ }^{2}}+\sum_{y_{1}, y_{2}=-\infty}^{\infty} q^{y_{1}{ }^{2}+y_{1}+3 y_{2}{ }^{2}+3 y_{2}} \\
= & \varphi(q) \varphi\left(q^{3}\right)+4 q \psi\left(q^{2}\right) \psi\left(q^{6}\right) .
\end{aligned}
$$

For the general case, we consider $\sum_{x_{1}, x_{2}=-\infty}^{\infty} q^{a x_{1}{ }^{2}+b x_{1} x_{2}+c x_{2}{ }^{2}}$, where $a, b, c$ are positive integers. We choose the matrix $B$ in the ECS such that the coefficients of $y_{1} y_{2}$ in $a\left(b_{11} y_{1}+b_{12} y_{2}\right)^{2}+b\left(b_{11} y_{1}+b_{12} y_{2}\right)\left(b_{21} y_{1}+b_{22} y_{2}\right)+c\left(b_{21} y_{1}+b_{22} y_{2}\right)^{2}$ to be zero. So the entries of the matrix $B$ must satisfy the requirement

$$
2 a b_{11} b_{12}+b\left(b_{11} b_{22}+b_{12} b_{21}\right)+2 c b_{21} b_{22}=0 \text {. }
$$

Accordingly, we prove an identity in [30] by L.-C. Shen. 


\section{Corollary 2.22.}

$$
\left(q^{2} ; q^{2}\right)_{\infty}^{2}=\sum_{m=-\infty}^{\infty} \sum_{n=-\infty}^{\infty}(-1)^{m} q^{2 m^{2}+2 m n+2 n^{2}+n} .
$$

Proof. This identity is equivalent to

$$
\left(q^{2} ; q^{2}\right)_{\infty}^{2}=\psi(-q)\left\{f\left(-q^{5},-q^{7}\right)-q f\left(-q,-q^{11}\right)\right\}
$$

if we choose matrix

$$
B=\left(\begin{array}{rr}
1 & -1 \\
1 & 1
\end{array}\right)
$$

By (1.11), $f\left(-q, q^{2}\right)=f\left(-q^{5},-q^{7}\right)-q f\left(-q,-q^{11}\right)$. So we only need to show that $\left(q^{2} ; q^{2}\right)_{\infty}^{2}=\psi(-q) f\left(-q, q^{2}\right)$. Recall an identity in [4, p. 11]

$$
\psi(q)=\frac{\left(q^{2}, q^{2}\right)_{\infty}}{\left(q ; q^{2}\right)_{\infty}}
$$

Noticing that

$$
\frac{\left(q^{2} ; q^{2}\right)_{\infty}}{\left(q ; q^{2}\right)_{\infty}}=\frac{\left(q^{2} ; q^{2}\right)_{\infty}^{2}}{(q ; q)_{\infty}}
$$

we replace $q$ with $-q$ in (2.65) and finish the proof.

\section{Products of Three or More Theta Functions}

We consider

$$
B=\left[\begin{array}{rrr}
1 & 1 & 1 \\
2 & -1 & 0 \\
1 & 1 & -1
\end{array}\right]
$$

The set of the 3 columns of $B$ is an orthogonal set and $\operatorname{det} B=6$.

Since

$$
B^{*}=\left[\begin{array}{rrr}
1 & 2 & 1 \\
2 & -2 & 2 \\
3 & 0 & -3
\end{array}\right]
$$

by Theorem 1.4, the integer matrix ECS $\left\{B \mathbb{Z}^{3}+\left(\begin{array}{l}r \\ 0 \\ 0\end{array}\right)\right\}_{r=0}^{5}$ covers $\mathbb{Z}^{3}$.

Consider

$$
S:=\prod_{i=1}^{3} f\left(a_{i}, b_{i}\right)=\sum_{x_{1}, x_{2}, x_{3}=-\infty}^{\infty} \prod_{i=1}^{3} a_{i}^{\frac{x_{i}{ }^{2}+x_{i}}{2}} b_{i}{ }^{\frac{x_{i}{ }^{2}-x_{i}}{2}} .
$$

Corresponding to the above ECS, we conclude the following special case of Theorem 1.4.

Corollary 3.1. For $q=a_{1} b_{1}=a_{2} b_{2}=a_{3} b_{3}$,

$$
\prod_{i=1}^{3} f\left(a_{i}, b_{i}\right)=f\left(a_{1} a_{2}{ }^{2} a_{3} q, b_{1} b_{2}{ }^{2} b_{3} q\right) f\left(a_{1} b_{2} a_{3}, b_{1} a_{2} b_{3}\right) f\left(a_{1} b_{3}, b_{1} a_{3}\right)
$$




$$
\begin{aligned}
& +a_{1} f\left(a_{1} a_{2}^{2} a_{3} q^{2}, b_{1} b_{2}^{2} b_{3}\right) f\left(a_{1} b_{2} a_{3} q, b_{1} a_{2} b_{3} / q\right) f\left(a_{1} b_{3} q, b_{1} a_{3} / q\right) \\
& +a_{2} f\left(a_{1} a_{2}^{2} a_{3} q^{3}, b_{1} b_{2}^{2} b_{3} / q\right) f\left(a_{1} b_{2} a_{3} / q, b_{1} a_{2} b_{3} q\right) f\left(a_{1} b_{3}, b_{1} a_{3}\right) \\
& +a_{1} a_{2} f\left(a_{1} a_{2}^{2} a_{3} q^{4}, b_{1} b_{2}{ }^{2} b_{3} / q^{2}\right) f\left(a_{1} b_{2} a_{3}, b_{1} a_{2} b_{3}\right) f\left(a_{1} b_{3} q, b_{1} a_{3} / q\right) \\
& +a_{1} a_{2} a_{3} f\left(a_{1} a_{2}{ }^{2} a_{3} q^{5}, b_{1} b_{2}{ }^{2} b_{3} / q^{3}\right) f\left(a_{1} b_{2} a_{3} q, b_{1} a_{2} b_{3} / q\right) f\left(a_{1} b_{3}, b_{1} a_{3}\right) \\
& +b_{3} f\left(a_{1} a_{2}{ }^{2} a_{3}, b_{1} b_{2}{ }^{2} b_{3} q^{2}\right) f\left(a_{1} b_{2} a_{3} / q, b_{1} a_{2} b_{3} q\right) f\left(a_{1} b_{3} q, b_{1} a_{3} / q\right) .
\end{aligned}
$$

If we begin with the solution set of the system of congruences, we need consider $B^{\prime}=6 B$. It is easy to verify that $\operatorname{det} B^{\prime}=1296=36^{3-1}$ and $36 \cdot B^{\prime-1}$ is an integer matrix. So $k^{\prime}=36$. Now $B^{\prime} y \equiv 0(\bmod 36)$ is equivalent to $B y \equiv 0(\bmod 6)$. By changing variables from $x_{i}$ to $y_{i}$ in $S$ by the transformation $y=A x$, where

$$
A=\left[\begin{array}{rrr}
1 & 2 & 1 \\
2 & -2 & 2 \\
3 & 0 & -3
\end{array}\right]
$$

we have $B y \equiv 0(\bmod 6)$. So for the system of congruences

$$
\left\{\begin{aligned}
y_{1}+y_{2}+y_{3} & \equiv 0(\bmod 6) \\
2 y_{1}-y_{2} & \equiv 0(\bmod 6) \\
y_{1}+y_{2}-y_{3} & \equiv 0(\bmod 6)
\end{aligned}\right.
$$

the solution set has six solutions modulo 6. We can also derive (3.2) by adding up the contribution of each solution to the sum $S$.

We can rewrite (3.2) as follows. For $a_{1}, a_{2}, a_{3} \neq 0$,

$$
\begin{aligned}
& \prod_{i=1}^{3}\left\langle-a_{i} ; q\right\rangle_{\infty} \\
= & \left\langle-a_{1} a_{2}{ }^{2} a_{3} q ; q^{6}\right\rangle_{\infty}\left\langle-a_{1} a_{2}{ }^{-1} a_{3} q ; q^{3}\right\rangle_{\infty}\left\langle-a_{1} a_{3}{ }^{-1} q ; q^{2}\right\rangle_{\infty} \\
& +a_{1}\left\langle-a_{1} a_{2}{ }^{2} a_{3} q^{2} ; q^{6}\right\rangle_{\infty}\left\langle-a_{1} a_{2}{ }^{-1} a_{3} q^{2} ; q^{3}\right\rangle_{\infty}\left\langle-a_{1} a_{3}{ }^{-1} q^{2} ; q^{2}\right\rangle_{\infty} \\
& +a_{2}\left\langle-a_{1} a_{2}{ }^{2} a_{3} q^{3} ; q^{6}\right\rangle_{\infty}\left\langle-a_{1} a_{2}{ }^{-1} a_{3} ; q^{3}\right\rangle_{\infty}\left\langle-a_{1} a_{3}{ }^{-1} q ; q^{2}\right\rangle_{\infty} \\
& +a_{1} a_{2}\left\langle-a_{1} a_{2}{ }^{2} a_{3} q^{4} ; q^{6}\right\rangle_{\infty}\left\langle-a_{1} a_{2}{ }^{-1} a_{3} q ; q^{3}\right\rangle_{\infty}\left\langle-a_{1} a_{3}{ }^{-1} q^{2} ; q^{2}\right\rangle_{\infty} \\
& +a_{1} a_{2} a_{3}\left\langle-a_{1} a_{2}{ }^{2} a_{3} q^{5} ; q^{6}\right\rangle_{\infty}\left\langle-a_{1} a_{2}{ }^{-1} a_{3} q^{2} ; q^{3}\right\rangle_{\infty}\left\langle-a_{1} a_{3}{ }^{-1} q ; q^{2}\right\rangle_{\infty} \\
& +a_{3}{ }^{-1} q\left\langle-a_{1} a_{2}{ }^{2} a_{3} ; q^{6}\right\rangle_{\infty}\left\langle-a_{1} a_{2}{ }^{-1} a_{3} ; q^{3}\right\rangle_{\infty}\left\langle-a_{1} a_{3}{ }^{-1} q^{2} ; q^{2}\right\rangle_{\infty} .
\end{aligned}
$$

Replacing $q$ with $q^{2}$, and then letting $a_{1}=b_{1}=a_{2}=b_{2}=a_{3}=b_{3}=q$ in (3.1), we deduce the following corollary.

\section{Corollary 3.2.}

$$
\begin{aligned}
\varphi^{3}(q)= & \varphi\left(q^{2}\right) \varphi\left(q^{3}\right) \varphi\left(q^{6}\right)+4 q \psi\left(q^{4}\right) f\left(q, q^{5}\right) f\left(q^{4}, q^{8}\right) \\
& +2 q \varphi\left(q^{2}\right) f\left(q, q^{5}\right) f\left(q^{2}, q^{10}\right)+4 q^{2} \varphi\left(q^{3}\right) \psi\left(q^{4}\right) \psi\left(q^{12}\right) .
\end{aligned}
$$

Letting $a_{1}=a_{2}=a_{3}=1, b_{1}=b_{2}=b_{3}=q$ in (3.1), we deduce the next corollary.

\section{Corollary 3.3.}

$$
4 \psi^{3}(q)=\phi(q) f\left(q, q^{2}\right) f\left(q, q^{5}\right)+2 \psi\left(q^{2}\right) f\left(q, q^{2}\right) f\left(q^{2}, q^{4}\right)
$$




$$
+\varphi(q) \varphi\left(q^{3}\right) \psi\left(q^{3}\right)+4 q \psi\left(q^{2}\right) \psi\left(q^{3}\right) \psi\left(q^{6}\right) .
$$

Letting $a_{3}=-a_{1}$ in (3.2), we deduce Corollary 2.9 after simplification.

Replacing each $a_{i}$ with $-a_{i}$, then replacing $a_{1}$ with $a, a_{2}$ with $a b$, and $a_{3}$ with $b$ in (3.2) and simplifying, we deduce the following analogue of Winquist's identity.

Corollary 3.4. For a,b nonzero,

$$
\begin{aligned}
& \left(a, a^{-1} q, b, b^{-1} q, a b, a^{-1} b^{-1} q, q, q ; q\right)_{\infty}^{2} \\
= & \left\langle-a b^{-1} q ; q^{2}\right\rangle_{\infty}\left[\left\langle-a^{3} b^{3} q ; q^{6}\right\rangle_{\infty}-a^{2} b^{2}\left\langle-a^{3} b^{3} q^{5} ; q^{6}\right\rangle_{\infty}\right] \\
& +\left\langle-a b^{-1} q^{2} ; q^{2}\right\rangle_{\infty}\left[a^{2} b\left\langle-a^{3} b^{3} q^{4} ; q^{6}\right\rangle_{\infty}-a\left\langle-a^{3} b^{3} q^{2} ; q^{6}\right\rangle_{\infty}\right] .
\end{aligned}
$$

The product in Corollary 3.4 is the product that occurs in MacDonald's identities [28] for $A_{2}$.

Recently, various representations of $(q ; q)_{\infty}^{10}$ have been given in [5], [27], [13], [8], [10], and [15. They are used in the proofs of Ramanujan's congruence $p(11 n+6) \equiv 0$ $(\bmod 11)$. In [9], S. Cooper, H-H. Chan, and P.C. Toh gave two representations of $(q ; q)_{\infty}^{8}$. Here we derive a new expression of $(q ; q)_{\infty}^{8}$.

Corollary 3.5.

$\begin{aligned} 16(q ; q)_{\infty}^{8}= & \sum_{m, n=-\infty}^{\infty}\left[(2 n-6 m+1)(2 n+6 m-2)^{2} q^{-2 m}-(2 n-6 m-3)(2 n+6 m+2)^{2} q^{2 m}\right. \\ & \left.+(2 n-6 m-1)(2 n+6 m+2)^{2} q^{n+m}-(2 n-6 m+1)(2 n+6 m)^{2} q^{n-m}\right] q^{n^{2}+3 m^{2}} .\end{aligned}$

Proof. Replacing $a$ with $a^{2}, b$ with $b^{2}$, and multiplying both sides of Corollary 3.4 by $a^{-1} b^{-1}$, we obtain

$$
\begin{aligned}
& (a-1 / a)(b-1 / b)\left(a^{2} q ; q\right)_{\infty}\left(a^{-2} q ; q\right)_{\infty}\left(b^{2} q ; q\right)_{\infty}\left(b^{-2} q ; q\right)_{\infty}\left(a^{2} b^{2} ; q\right)_{\infty}\left(a^{-2} b^{-2} q ; q\right)_{\infty}(q ; q)_{\infty}^{2} \\
= & \sum_{m, n=-\infty}^{\infty}\left[a^{2 n+6 m-1} b^{-2 n+6 m-1} q^{-2 m}-a^{2 n+6 m+3} b^{-2 n+6 m+3} q^{2 m}\right. \\
& \left.+a^{2 n+6 m+3} b^{-2 n+6 m+1} q^{n+m}-a^{2 n+6 m+1} b^{-2 n+6 m-1} q^{n-m}\right] q^{n^{2}+3 m^{2}} .
\end{aligned}
$$

We differentiate (3.5) with respect to $b$, let $b=1$, and then multiply both sides by $a^{-1}$ to obtain

$$
\begin{aligned}
& -2(a-1 / a)^{2}\left(a^{2} q ; q\right)_{\infty}^{2}\left(a^{-2} q ; q\right)_{\infty}^{2}(q ; q)_{\infty}^{4} \\
= & \sum_{m, n=-\infty}^{\infty}\left[(-2 n+6 m-1) a^{2 n+6 m-2} q^{-2 m}-(-2 n+6 m+3) a^{2 n+6 m+2} q^{2 m}\right. \\
& \left.+(-2 n+6 m+1) a^{2 n+6 m+2} q^{n+m}-(-2 n+6 m-1) a^{2 n+6 m} q^{n-m}\right] q^{n^{2}+3 m^{2}} .
\end{aligned}
$$

Applying the differential operator $a \frac{d}{d a}$ twice, and letting $a=1$ in (3.6), we deduce Corollary 3.5. 
Replacing $q$ by $q^{5}$ in (3.3), and then letting $a=q, b=q^{3}$, replacing $q$ by $q^{5}$ in (3.3), and then choosing $a=q, b=q^{2}$, we deduce the following results.

\section{Corollary 3.6.}

$$
\begin{aligned}
f(-q) f\left(-q,-q^{4}\right) & =f\left(q^{3}, q^{7}\right)\left[f\left(q^{13}, q^{17}\right)-q f\left(q^{7}, q^{23}\right)\right\}+f\left(q^{2}, q^{8}\right)\left\{q^{3} f\left(q^{2}, q^{28}\right)-q f\left(q^{8}, q^{22}\right)\right] . \\
f(-q) f\left(-q^{2},-q^{3}\right) & =f\left(q^{4}, q^{6}\right)\left[f\left(q^{14}, q^{16}\right)-q^{2} f\left(q^{4}, q^{26}\right)\right\}+f\left(q, q^{9}\right)\left\{q^{4} f\left(q, q^{29}\right)-q f\left(q^{11}, q^{19}\right)\right] .
\end{aligned}
$$

Letting $b=-1$ in (3.3), we deduce the next corollary.

Corollary 3.7. For $a, b$ nonzero,

$$
\begin{aligned}
& 2\left\langle a^{2} ; q^{2}\right\rangle_{\infty} \\
= & \left(a q, a^{-1} q ; q^{2}\right)_{\infty}\left[\left\langle a^{3} q ; q^{6}\right\rangle_{\infty}-a^{2}\left\langle a^{3} q^{5} ; q^{6}\right\rangle_{\infty}\right]-\left(a q^{2}, a^{-1} ; q^{2}\right)_{\infty}\left[a\left\langle a^{3} q^{2} ; q^{6}\right\rangle_{\infty}+a^{2}\left\langle a^{3} q^{4} ; q^{6}\right\rangle_{\infty}\right] .
\end{aligned}
$$

Letting $b=-a$ in (3.3), we deduce the next corollary.

Corollary 3.8. For nonzero complex number a,

$$
\left\langle a^{2} ; q^{2}\right\rangle_{\infty}\left(-a^{2},-a^{-2} q ; q\right)_{\infty}=\left\langle a^{6} q ; q^{6}\right\rangle_{\infty}-a^{4}\left\langle a^{6} q^{5} ; q^{6}\right\rangle_{\infty}
$$

Letting $b=1$ in (3.3), we deduce the next corollary.

Corollary 3.9. For nonzero complex number a,

$$
\begin{aligned}
& \left\langle-a q ; q^{2}\right\rangle_{\infty}\left[\left\langle-a^{3} q ; q^{6}\right\rangle_{\infty}-a^{2}\left\langle-a^{3} q^{5} ; q^{6}\right\rangle_{\infty}\right] \\
+ & \left\langle-a q^{2} ; q^{2}\right\rangle_{\infty}\left[\left\langle a^{2}\left(-a^{3} q^{4} ; q^{6}\right\rangle_{\infty}-a\left\langle-a^{3} q^{2} ; q^{6}\right\rangle_{\infty}\right]=0 .\right.
\end{aligned}
$$

The next simplest orthogonal matrix with $l_{1}=l_{2}=l_{3}$ is

$$
B=\left[\begin{array}{rrr}
1 & 1 & 3 \\
1 & -1 & -3 \\
0 & 3 & -2
\end{array}\right]
$$

with $\operatorname{det} B=22$. We skip the identity generated by this matrix here.

If we do not require that all the $l_{i}$ are the same, we can obtain other "generalized orthogonal" matrices. For example, if we choose $l_{1}=l_{2}=1, l_{3}=2$, then for the following "generalized orthogonal" matrix

$$
B=\left[\begin{array}{rrr}
1 & 1 & 1 \\
1 & -1 & 1 \\
1 & 0 & -1
\end{array}\right],
$$

since det $B=4$, we can write a product of three theta functions as the linear combination of four products of three theta functions. Omitting the details, we deduce the following corollary.

Corollary 3.10. For $a_{1} b_{1}=a_{2} b_{2}=q$ and $a_{3} b_{3}=q^{2}$,

$$
\begin{aligned}
& \prod_{i=1}^{3} f\left(a_{i}, b_{i}\right) \\
= & f\left(a_{1} b_{2}, b_{1} a_{2}\right)
\end{aligned}
$$




$$
\begin{aligned}
& \times\left[f\left(a_{1} a_{2} a_{3}, b_{1} b_{2} b_{3}\right) f\left(a_{1} a_{2} b_{3}, b_{1} b_{2} a_{3}\right)+a_{1} a_{2} f\left(a_{1} a_{2} a_{3} q^{2}, b_{1} b_{2} b_{3} / q^{2}\right) f\left(a_{1} a_{2} b_{3} q^{2}, b_{1} b_{2} a_{3} / q^{2}\right)\right] \\
& +a_{1} f\left(a_{1} b_{2} q, b_{1} a_{2} / q\right) \\
& \times\left[f\left(a_{1} a_{2} a_{3} q, b_{1} b_{2} b_{3} / q\right) f\left(a_{1} a_{2} b_{3} q, b_{1} b_{2} a_{3} / q\right)+a_{3} f\left(a_{1} a_{2} a_{3} q^{3}, b_{1} b_{2} b_{3} / q^{3}\right) f\left(a_{1} a_{2} b_{3} / q, b_{1} b_{2} a_{3} q\right)\right] .
\end{aligned}
$$

We can rewrite the above identity. For $a_{1}, a_{2}, a_{3} \neq 0$,

$$
\begin{aligned}
& \left\langle-a_{1} ; q\right\rangle_{\infty}\left\langle-a_{2} ; q\right\rangle_{\infty}\left\langle-a_{3} ; q^{2}\right\rangle_{\infty} \\
= & \left\langle-a_{1} a_{2}{ }^{-1} q ; q^{2}\right\rangle_{\infty} \\
& \times\left[\left\langle-a_{1} a_{2} a_{3} ; q^{4}\right\rangle_{\infty}\left\langle-a_{1} a_{2} a_{3}{ }^{-1} q^{2} ; q^{4}\right\rangle_{\infty}+a_{1} a_{2}\left\langle-a_{1} a_{2} a_{3} q^{2} ; q^{4}\right\rangle_{\infty}\left\langle-a_{1} a_{2} a_{3}{ }^{-1} q^{4} ; q^{4}\right\rangle_{\infty}\right] \\
& +a_{1}\left\langle-a_{1} a_{2}{ }^{-1} q^{2} ; q^{2}\right\rangle_{\infty} \\
& \times\left[\left\langle-a_{1} a_{2} a_{3} q ; q^{4}\right\rangle_{\infty}\left\langle-a_{1} a_{2} a_{3}{ }^{-1} q^{3} ; q^{4}\right\rangle_{\infty}+a_{3}\left\langle-a_{1} a_{2} a_{3} q^{3} ; q^{4}\right\rangle_{\infty}\left\langle-a_{1} a_{2} a_{3}{ }^{-1} q ; q^{4}\right\rangle_{\infty}\right] .
\end{aligned}
$$

Letting $a_{3}=-a_{1} a_{2}$ in Corollary 3.10 , then replacing $a_{1}$ with $-a_{1}, a_{2}$ with $-a_{2}$, and simplifying, we deduce the next corollary.

\section{Corollary 3.11.}

$$
\begin{aligned}
& \left\langle a_{1} ; q\right\rangle_{\infty}\left\langle a_{2} ; q\right\rangle_{\infty}\left\langle a_{1} a_{2} ; q^{2}\right\rangle_{\infty} \\
= & \left\langle q^{2} ; q^{4}\right\rangle_{\infty}\left\langle-a_{1} a_{2}{ }^{-1} q ; q^{2}\right\rangle_{\infty}\left\langle a_{1}{ }^{2} a_{2}{ }^{2} ; q^{4}\right\rangle_{\infty} \\
& +a_{1}\left\langle q ; q^{4}\right\rangle_{\infty}\left\langle-a_{1} a_{2}{ }^{-1} q^{2} ; q^{2}\right\rangle_{\infty}\left[a_{1} a_{2}\left\langle a_{1}{ }^{2} a_{2}{ }^{2} q^{3} ; q^{4}\right\rangle_{\infty}-\left\langle a_{1}{ }^{2} a_{2}{ }^{2} q ; q^{4}\right\rangle_{\infty}\right] .
\end{aligned}
$$

Letting $a_{2}=-1$ in Corollary 3.11 and change the notation from $a_{1}$ to $a$, we deduce the next result.

\section{Corollary 3.12.}

$$
\begin{aligned}
& 2(a ; q)_{\infty}\left(a^{-1} q ; q\right)_{\infty}\left(-a ; q^{2}\right)_{\infty}\left(-a^{-1} q^{2} ; q^{2}\right)_{\infty} \\
= & \left(a^{2} ; q^{4}\right)_{\infty}\left(a^{-2} q^{4} ; q^{4}\right)_{\infty}\left(a q ; q^{2}\right)_{\infty}\left(a^{-1} q ; q^{2}\right)_{\infty} \\
& -\left(a q^{2} ; q^{2}\right)_{\infty}\left(a^{-1} ; q^{2}\right)_{\infty}\left(q ; q^{2}\right)_{\infty}\left(-q^{2}, q^{2}\right)_{\infty}^{2} \\
& \times\left[a^{2}\left(a^{2} q^{3} ; q^{4}\right)_{\infty}\left(a^{-2} q ; q^{4}\right)_{\infty}+a\left(a^{2} q ; q^{4}\right)_{\infty}\left(a^{-2} q^{3} ; q^{4}\right)_{\infty}\right] .
\end{aligned}
$$

Next, we study the transformation matrix

$$
B=\left[\begin{array}{rrrr}
1 & 1 & 0 & -1 \\
1 & -1 & 0 & 1 \\
0 & 1 & -1 & 1 \\
0 & 1 & 1 & 1
\end{array}\right]
$$

The set of its four columns of $B$ is an orthogonal set and $\operatorname{det} B=8$. Foregoing all details, we have the following corollary.

Corollary 3.13. If we let $q=a_{i} b_{i}$, where $i=1,2,3,4$, then

$$
\prod_{i=1}^{4} f\left(a_{i}, b_{i}\right)=\sum_{r=0}^{7} a_{1} \frac{r^{2}+r}{2} b_{1} \frac{r^{2}-r}{2} f\left(a_{1} a_{2} q^{r}, b_{1} b_{2} q^{-r}\right) f\left(a_{1} b_{2} a_{3} a_{4} q^{r}, b_{1} a_{2} b_{3} b_{4} q^{-r}\right)
$$




$$
\times f\left(b_{3} a_{4} q^{r}, a_{3} b_{4} q^{-r}\right) f\left(b_{1} a_{2} a_{3} a_{4} q^{r}, a_{1} b_{2} b_{3} b_{4} q^{-r}\right) .
$$

Corollary 3.10 can be shown as a special case of Corollary 3.13. We omit the details here.

Corollary 3.13 can be rewritten as follows. For $a_{1}, a_{2}, a_{3}, a_{4} \neq 0$,

$$
\begin{aligned}
& \prod_{i=1}^{4}\left\langle-a_{i} ; q\right\rangle_{\infty} \\
= & \left\langle-a_{1} a_{2} ; q^{2}\right\rangle_{\infty}\left\langle-a_{1} a_{2}{ }^{-1} a_{3} a_{4} q ; q^{4}\right\rangle_{\infty}\left\langle-a_{3}{ }^{-1} a_{4} q ; q^{2}\right\rangle_{\infty}\left\langle-a_{1}^{-1} a_{2} a_{3} a_{4} q ; q^{4}\right\rangle_{\infty} \\
& +a_{1}\left\langle-a_{1} a_{2} q ; q^{2}\right\rangle_{\infty}\left\langle-a_{1} a_{2}{ }^{-1} a_{3} a_{4} q^{2} ; q^{4}\right\rangle_{\infty}\left\langle-a_{3}{ }^{-1} a_{4} q ; q^{2}\right\rangle_{\infty}\left\langle-a_{1}^{-1} a_{2} a_{3} a_{4} ; q^{4}\right\rangle_{\infty} \\
& +a_{2}\left\langle-a_{1} a_{2} q ; q^{2}\right\rangle_{\infty}\left\langle-a_{1} a_{2}{ }^{-1} a_{3} a_{4} ; q^{4}\right\rangle_{\infty}\left\langle-a_{3}{ }^{-1} a_{4} q ; q^{2}\right\rangle_{\infty}\left\langle-a_{1}^{-1} a_{2} a_{3} a_{4} q^{2} ; q^{4}\right\rangle_{\infty} \\
& +a_{3}\left\langle-a_{1} a_{2} ; q^{2}\right\rangle_{\infty}\left(-a_{1} a_{2}{ }^{-1} a_{3} a_{4} q^{2} ; q^{4}\right\rangle_{\infty}\left(-a_{3}{ }^{-1} a_{4} ; q^{2}\right)_{\infty}\left(-a_{1}^{-1} a_{2} a_{3} a_{4} q^{2} ; q^{4}\right)_{\infty} \\
& +a_{1} a_{3}\left\langle-a_{1} a_{2} q ; q^{2}\right\rangle_{\infty}\left\langle-a_{1} a_{2}{ }^{-1} a_{3} a_{4} q^{3} ; q^{4}\right\rangle_{\infty}\left\langle-a_{3}{ }^{-1} a_{4} ; q^{2}\right\rangle_{\infty}\left\langle-a_{1}^{-1} a_{2} a_{3} a_{4} q ; q^{4}\right\rangle_{\infty} \\
& +a_{2} a_{3}\left\langle-a_{1} a_{2} q ; q^{2}\right\rangle_{\infty}\left\langle-a_{1} a_{2}{ }^{-1} a_{3} a_{4} q ; q^{4}\right\rangle_{\infty}\left\langle-a_{3}{ }^{-1} a_{4} ; q^{2}\right\rangle_{\infty}\left\langle-a_{1}^{-1} a_{2} a_{3} a_{4} q^{3} ; q^{4}\right\rangle_{\infty} \\
& +a_{3} a_{4}\left\langle-a_{1} a_{2} ; q^{2}\right\rangle_{\infty}\left\langle-a_{1} a_{2}{ }^{-1} a_{3} a_{4} q^{3} ; q^{4}\right\rangle_{\infty}\left\langle-a_{3}{ }^{-1} a_{4} q ; q^{2}\right\rangle_{\infty}\left\langle-a_{1}^{-1} a_{2} a_{3} a_{4} q^{3} ; q^{4}\right\rangle_{\infty} \\
& +a_{4}{ }^{-1} q\left\langle-a_{1} a_{2} ; q^{2}\right\rangle_{\infty}\left\langle-a_{1} a_{2}{ }^{-1} a_{3} a_{4} q ; q^{4}\right\rangle_{\infty}\left\langle-a_{3}{ }^{-1} a_{4} q ; q^{2}\right\rangle_{\infty}\left\langle-a_{1}^{-1} a_{2} a_{3} a_{4} q ; q^{4}\right\rangle_{\infty} .
\end{aligned}
$$

From (3.15), we deduce the following.

Corollary 3.14. Let $q=a_{i} b_{i},(i=1,2,3,4)$, then

$$
\begin{aligned}
& \prod_{i=1}^{4} f\left(a_{i}, b_{i}\right)+\prod_{i=1}^{4} f\left(-a_{i},-b_{i}\right) \\
= & 2 f\left(a_{1} a_{2}, b_{1} b_{2}\right) f\left(a_{1} b_{2} a_{3} a_{4}, b_{1} a_{2} b_{3} b_{4}\right) f\left(a_{3} b_{4}, a_{4} b_{3}\right) f\left(a_{1} b_{2} b_{3} b_{4}, b_{1} a_{2} a_{3} a_{4}\right) \\
& +2 a_{1} a_{4} f\left(a_{1} a_{2} q, b_{1} b_{2} / q\right) f\left(a_{1} b_{2} a_{3} a_{4} q^{2}, b_{1} a_{2} b_{3} b_{4} / q^{2}\right) f\left(a_{3} b_{4} / q, a_{4} b_{3} q\right) f\left(a_{1} b_{2} b_{3} b_{4}, b_{1} a_{2} a_{3} a_{4}\right) \\
& +2 a_{2} a_{4} f\left(a_{1} a_{2} q, b_{1} b_{2} / q\right) f\left(a_{1} b_{2} a_{3} a_{4}, b_{1} a_{2} b_{3} b_{4}\right) f\left(a_{3} b_{4} / q, a_{4} b_{3} q\right) f\left(a_{1} b_{2} b_{3} b_{4} / q^{2}, b_{1} a_{2} a_{3} a_{4} q^{2}\right) \\
& +2 a_{3} a_{4} f\left(a_{1} a_{2}, b_{1} b_{2}\right) f\left(a_{1} b_{2} a_{3} a_{4} q^{2}, b_{1} a_{2} b_{3} b_{4} / q^{2}\right) f\left(a_{3} b_{4}, a_{4} b_{3}\right) f\left(a_{1} b_{2} b_{3} b_{4} / q^{2}, b_{1} a_{2} a_{3} a_{4} q^{2}\right) . \\
& \prod_{i=1}^{4} f\left(a_{i}, b_{i}\right)-\prod_{i=1}^{4} f\left(-a_{i},-b_{i}\right) \\
= & 2 a_{1} f\left(a_{1} a_{2} q, b_{1} b_{2} / q\right) f\left(a_{1} b_{2} a_{3} a_{4} q, b_{1} a_{2} b_{3} b_{4} / q\right) f\left(a_{3} b_{4}, a_{4} b_{3}\right) f\left(a_{1} b_{2} b_{3} b_{4} q, b_{1} a_{2} a_{3} a_{4} / q\right) \\
& +2 a_{2} f\left(a_{1} a_{2} q, b_{1} b_{2} / q\right) f\left(a_{1} b_{2} a_{3} a_{4} / q, b_{1} a_{2} b_{3} b_{4} q\right) f\left(a_{3} b_{4}, a_{4} b_{3}\right) f\left(a_{1} b_{2} b_{3} b_{4} / q, b_{1} a_{2} a_{3} a_{4} q\right) \\
& +2 a_{4} f\left(a_{1} a_{2}, b_{1} b_{2}\right) f\left(a_{1} b_{2} a_{3} a_{4} q, b_{1} a_{2} b_{3} b_{4} / q\right) f\left(a_{3} b_{4} / q, a_{4} b_{3} q\right) f\left(a_{1} b_{2} b_{3} b_{4} / q, b_{1} a_{2} a_{3} a_{4} q\right) \\
& +2 b_{3} f\left(a_{1} a_{2}, b_{1} b_{2}\right) f\left(a_{1} b_{2} a_{3} a_{4} / q, b_{1} a_{2} b_{3} b_{4} q\right) f\left(a_{3} b_{4} / q, a_{4} b_{3} q\right) f\left(a_{1} b_{2} b_{3} b_{4} q, b_{1} a_{2} a_{3} a_{4} / q\right) .
\end{aligned}
$$

M. Hirschhorn [22] considered the transformation matrix

$$
A=\left[\begin{array}{rrrr}
1 & 0 & 1 & 1 \\
0 & 1 & 1 & -1 \\
1 & 1 & -1 & 0 \\
1 & -1 & 0 & -1
\end{array}\right]
$$


and derived a generalization of Winquist's identity.

If we consider the transformation matrix as a Hadamard matrix with determinant 16

$$
B=\left[\begin{array}{rrrr}
1 & 1 & 1 & 1 \\
1 & 1 & -1 & -1 \\
1 & -1 & -1 & 1 \\
1 & -1 & 1 & -1
\end{array}\right]
$$

we deduce the following corollary corresponding to its ECS.

Corollary 3.15. Let $q=a_{i} b_{i}$, where $i=1,2,3,4$, then

$$
\begin{aligned}
\prod_{i=1}^{4} f\left(a_{i}, b_{i}\right)= & \sum_{r=0}^{15} a_{1} \frac{r^{2}+r}{2} b_{1} \frac{r^{2}-r}{2} f\left(a_{1} a_{2} a_{3} a_{4} q^{r}, b_{1} b_{2} b_{3} b_{4} q^{-r}\right) f\left(a_{1} a_{2} b_{3} b_{4} q^{r}, b_{1} b_{2} a_{3} a_{4} q^{-r}\right) \\
& \times f\left(a_{1} b_{2} b_{3} a_{4} q^{r}, b_{1} a_{2} a_{3} b_{4} q^{-r}\right) f\left(a_{1} b_{2} a_{3} b_{4} q^{r}, b_{1} a_{2} b_{3} a_{4} q^{-r}\right) .
\end{aligned}
$$

Replacing $a_{3}$ with $a_{1}, a_{4}$ with $-a_{2}$, and then $a_{2}{ }^{2}$ with $a_{2}$ in (3.17), we deduce the following corollary.

\section{Corollary 3.16.}

$$
\begin{aligned}
& \left\langle-a_{1} ; q\right\rangle_{\infty}^{2}\left\langle-a_{2} ; q^{2}\right\rangle_{\infty} \\
= & \varphi(q)\left[\left\langle-a_{1}^{2} a_{2} ; q^{4}\right\rangle_{\infty}\left\langle-a_{1}^{2} a_{2}{ }^{-1} q^{2} ; q^{4}\right\rangle_{\infty}+a_{1}{ }^{2}\left\langle a_{1}{ }^{2} a_{2} q^{2} ; q^{4}\right\rangle_{\infty}\left\langle a_{1}{ }^{2} a_{2}{ }^{-1} q^{4} ; q^{4}\right\rangle_{\infty}\right] \\
& +2 \psi\left(q^{2}\right)\left[a_{1}\left\langle-a_{1}{ }^{2} a_{2} q ; q^{4}\right\rangle_{\infty}\left\langle a_{1}^{2} a_{2}{ }^{-1} q^{3} ; q^{4}\right\rangle_{\infty}+a_{1} a_{2}\left\langle-a_{1}{ }^{2} a_{2} q^{3} ; q^{4}\right\rangle_{\infty}\left\langle a_{1}{ }^{2} a_{2}{ }^{-1} q ; q^{4}\right\rangle_{\infty}\right] .
\end{aligned}
$$

We can rewrite (3.18) as follows. For $a b=q, c d=q^{2}$,

$$
\begin{aligned}
& f^{2}(a, b) f(c, d) \\
= & \varphi(q)\left[\left(a^{2} c, b^{2} d\right) f\left(a^{2} d, b^{2} c\right)+a^{2} f\left(a^{2} c q^{2}, b^{2} d / q^{2}\right) f\left(a^{2} d q^{2}, b^{2} c / q^{2}\right)\right] \\
& +2 \psi\left(q^{2}\right)\left[\left(a f\left(a^{2} c q, b^{2} d / q\right) f\left(a^{2} d q, b^{2} c / q\right)+b f\left(a^{2} c / q, b^{2} d q\right) f\left(a^{2} d / q, b^{2} c q\right)\right] .\right.
\end{aligned}
$$

In (3.19), setting $c=-a^{2}$ and $d=-b^{2}$. By (1.11), $f\left(a^{-1} b,-a^{2}\right)=f\left(-a^{-1} b^{3},-a^{5} b\right)+$ $a^{-1} b f\left(-a^{3} b^{-1},-a b^{5}\right)$. We deduce the following identity.

Corollary 3.17. For $a b=q$,

$$
f^{2}(a, b) f\left(-a^{2},-b^{2}\right)=\varphi(q) \varphi\left(-q^{2}\right) f\left(-a^{4},-b^{4}\right)+2 a \psi(-q) \psi\left(q^{2}\right) f\left(a^{-1} b,-a^{2}\right) .
$$

Similarly, setting $c=a^{2}$ and $d=b^{2}$ in (3.19), we have the next corollary after simplification.

Corollary 3.18. For $a b=q$,

$$
\begin{aligned}
& f^{2}(a, b) f\left(a^{2}, b^{2}\right) \\
= & \varphi(q)\left[f\left(a^{4}, b^{4}\right) \phi\left(a^{2} b^{2}\right)+2 a^{2} f\left(a^{-2} b^{2}, a^{4} q^{2}\right) \psi\left(q^{4}\right)\right]+2 a \psi(q) \psi\left(q^{2}\right) f\left(a^{-1} b, a^{2}\right) .
\end{aligned}
$$

Using (3.19), we deduce the following corollary. 
Corollary 3.19. For $a b=q, c d=q^{2}$,

$$
\begin{aligned}
& {\left[f^{2}(a, b)+f^{2}(-a,-b)\right] f(c, d) } \\
= & 2 \varphi(q)\left[f\left(a^{2} c, b^{2} d\right) f\left(a^{2} d, b^{2} c\right)+a^{2} f\left(a^{-2} d, a^{2} c q^{2}\right) f\left(a^{-2} c, a^{2} d q^{2}\right)\right] .
\end{aligned}
$$

We rewrite the above identity in its equivalent form.

$$
\begin{aligned}
& {\left[(a ; q)_{\infty}^{2}\left(a^{-1} q ; q\right)_{\infty}^{2}+(-a ; q)_{\infty}^{2}\left(-a^{-1} q ; q\right)_{\infty}^{2}\right]\left(b ; q^{2}\right)_{\infty}\left(b^{-1} q^{2} ; q^{2}\right)_{\infty} } \\
= & 2(-q ; q)_{\infty}^{4}\left(a^{2} b ; q^{4}\right)_{\infty}\left(a^{-2} b^{-1} q^{4} ; q^{4}\right)_{\infty}\left(a^{2} b^{-1} q^{2} ; q^{4}\right)_{\infty}\left(a^{-2} b q^{2} ; q^{4}\right)_{\infty} \\
& +2 a^{2}(-q ; q)_{\infty}^{4}\left(a^{2} b q^{2} ; q^{4}\right)_{\infty}\left(a^{-2} b^{-1} q^{2} ; q^{4}\right)_{\infty}\left(a^{2} b^{-1} q^{4} ; q^{4}\right)_{\infty}\left(a^{-2} b ; q^{4}\right)_{\infty} .
\end{aligned}
$$

Setting $b=a^{2}$ in (3.23), we deduce the following corollary after simplification.

\section{Corollary 3.20.}

$$
f^{2}(a, b)+f^{2}(-a,-b)=2 f\left(a^{2}, b^{2}\right) \varphi(a b) .
$$

This is (v) of Entry 30 in [2, p. 46].

We can rewrite the above identity as

$$
(a ; q)_{\infty}^{2}\left(a^{-1} q ; q\right)_{\infty}^{2}+(-a ; q)_{\infty}^{2}\left(-a^{-1} q ; q\right)_{\infty}^{2}=2(-q ; q)_{\infty}^{2}\left(-q ; q^{2}\right)_{\infty}^{2}\left(-a^{2} ; q^{2}\right)_{\infty}\left(-a^{-2} q^{2} ; q^{2}\right)_{\infty}
$$

We can find other interesting cases by exploring different integer matrix exact covering systems. For example, if we choose

$$
B=\left[\begin{array}{rrrrr}
1 & 0 & -1 & -1 & 1 \\
1 & 0 & 0 & 2 & 0 \\
0 & 1 & 1 & 0 & 1 \\
0 & -1 & 1 & 0 & 1 \\
-1 & 0 & -1 & 1 & 1
\end{array}\right]
$$

we can write a product of five theta functions as the linear combination of twenty four products of five theta functions. We omit the details here.

Acknowledgement: This work grow out of the author's Ph.D. dissertation. The author is very grateful to his advisor Professor Bruce Berndt for his helpful guidance.

\section{REFERENCES}

[1] N. D. Baruah and J. Bora, Modular relations for the nonic analogues of the Rogers-Ramanujan functions with applications to partitions, J. Number Theory. 128 (2008), no. 1, 175-206.

[2] B. C. Berndt, Ramanujan's notebooks, Part III, Springer-Verlag, New York, 1991.

[3] B. C. Berndt, Ramanujan's notebooks, Part IV, Springer-Verlag, New York, 1994.

[4] B. C. Berndt, Number theory in the spirit of Ramanujan, Student Mathematical Library, vol. 34, American Mathematical Society, Providence, RI, 2006.

[5] B. C. Berndt, S. H. Chan, Z.-G. Liu, and H. Yesilyurt, A new identity for $(q ; q)_{\infty}^{10}$ with an application to Ramanujan's partition congruence modulo 11, Q. J. Math. 55 (2004), no. 1, 13-30.

[6] R. Blecksmith, J. Brillhart, and I. Gerst, Some infinite product identities, Math. Comp. 51 (1988), no. $183,301-314$.

[7] J. M. Borwein and P. B. Borwein, Pi and the AGM: A study in analytic number theory and computational complexity, Wiley-Interscience, New York, 1987.

[8] H. H. Chan, Z.-G. Liu, and S. T. Ng, Elliptic functions and the quintuple, Hirschhorn and Winquist product identities, Int. J. Number Theory 1 (2005), no. 1, 33-43. 
[9] H. H. Chan, S. Cooper, and P. C. Toh, Ramanujan's Eisenstein series and powers of Dedekind's eta-function, J. London Math. Soc. 75 (2007), no. 1, 225-242.

[10] S. H. Chan, Generalized Lambert series identities, Proc. London Math. Soc. (3) 91 (2005), no. 3, $598-622$.

[11] S. H. Chan, A new proof of Winquist's identity, Proceedings of Ramanujan rediscovered conference, to appear.

[12] S. L. Chen and S.-S. Huang, New modular relations for the Göllnitz-Gordon functions, J. Number Theory. 93 (2002), no. 1, 58-75.

[13] W. Chu, Theta function identities and Ramanujan's congruences on the partition function, Q. J. Math. 56 (2005), no. 4, 491-506.

[14] W. Chu and Q. Yan, Unification of the quintuple and septuple product identities, Electron. J. Combin. 14 (2007), \#N7.

[15] W. Chu and Q. Yan, Winquist's identity and Ramanujan's partition congruence $p(11 n+6) \equiv 0$ (mod 11), European J. Combin. 29 (2008), no. 3, 581-591.

[16] S. Cooper, The quintuple product identity, Int. J. Number Theory. 2 (2006), no. 1, 115-161.

[17] J. A. Ewell, On an identity of a Ramanujan, Proc. Amer. Math. Soc. 125 (1997), 3769-3771.

[18] M. A. Fiol, Congruences in $\mathbb{Z}^{n}$, finite abelian groups and the chinese remainder theorem, Discrete Math. 67 (1987), no. 1, 101-105.

[19] G. Gasper and M. Rahman, Basic hypergeometric series, Cambridge University Press, Cambridge, 2004.

[20] H. Hahn, Septic analogues of the Rogers-Ramanujan functions, Acta Arith. 110 (2003), no. 4, 381-399.

[21] M. D. Hirschhorn, A simple proof of an identity of Ramanujan, J. Austral. Math. Soc. Ser. A 34 (1983), 31-35.

[22] M.D. Hirschhorn, A generalisation of Winquist's identity and a conjecture of Ramanujan, J. Indian Math. Soc. 51 (1987), 49-55.

[23] M. D. Hirschhorn, A generalization of the quintuple product identity, J. Austral. Math. Soc. Ser. A 44 (1988), no. 1, 42-45.

[24] S.-S. Huang, On modular relations for the Göllnitz-Gordon functions with applications to partitions, J. Number Theory. 68 (1998), no. 2, 178-216.

[25] S.-S. Huang, A note on Hirschhorn's generalization of the quintuple product identity, South East Asian J. Math. Math. Sci. 3 (2005), no. 2, 39-41.

[26] S. Kongsiriwong and Z.-G. Liu, Uniform proofs of q-series-product identities, Results Math. 44 (2003), no. 3-4, 312-339.

[27] Z.-G. Liu, A theta function identity and its implications, Trans. Amer. Math. Soc. 357 (2005), no. $2,825-835$.

[28] I. Macdonald, Affine root systems and Dedekind's $\eta$-function, Invent. Math. 15 (1972), 91-143.

[29] H. A. Schwarz, Formeln und Lehrsätze zum Gebrauche der Elliptischen Funktionen nach Vorlesungen und Aufzeichnungen des Herrn Prof. K. Weierstrass, Berlin, 1893.

[30] L.-C. Shen, On the products of three theta functions, The Ramanujan Journal 3 (1999), 343-357.

[31] L. J. Slater, Further identities of the Rogers-Ramanujan type, Proc. London Math. Soc.(2) 54 (1952), 147-167.

[32] S. H. Son, Some theta function identities related to the Rogers-Ramanujan continued fraction, Proc. Amer. Math. Soc. 126 (1998), no. 10, 2895-2902.

[33] J. Tannery and J. Molk, Élements de la theorie des fonctions elliptiques, Chelsea, New York, 1972.

[34] L. Winquist, An elementary proof of $p(11 n+6) \equiv 0(\bmod 11)$, J. Combin. Theory. 6 (1969), $56-59$.

Department of Mathematics, University of Mississippi, University, MS 38677, USA

E-mail address: zcao3@olemiss.edu 